\title{
A posteriori pointwise error computation for 2-D transport equations based on the variational multiscale method
}

\author{
Diego Irisarri, Guillermo Hauke \\ LIFTEC (CSIC) - Universidad de Zaragoza \\ Escuela de Ingeniería y Arquitectura, Área de Mecánica de Fluidos \\ C/María de Luna 3, 50018 Zaragoza, Spain \\ e-mail: dirisarri@unizar.es
}

\begin{abstract}
This article presents a general framework to estimate the pointwise error of linear partial differential equations. The error estimator is based on the variational multiscale theory, in which the error is decomposed in two components according to the nature of the residuals: element interior residuals and interelement jumps. The relationship between the residuals (coarse scales) and the error components (fine scales) is established, yielding to a very simple model. In particular, the pointwise error is modeled as a linear combination of bubble functions and Green's functions. If residual-free bubbles and the classical Green's function are employed, the technology leads to an exact explicit method for the pointwise error. If bubble functions and free-space Green's functions are employed, then a local projection problem must be solved within each element and a global boundary integral equation must be solved on the domain boundary. As a consequence, this gives a model for the so-called fine-scale Green's functions. The numerical error is studied for the standard Galerkin and SUPG methods with application to the heat equation, the reaction-diffusion equation and the convection-diffusion equation. Numerical results show that stabilized methods minimize the propagation of pollution errors, which stay mostly locally.
\end{abstract}

Keywords: A posteriori pointwise error estimation, finite elements, variational multiscale method, transport equation, Green's functions, residual-free bubbles 


\section{Introduction}

Finite Element methods (FEM), together with other numerical methods, are widely used owing to the increasing need of solving partial differential equations applied to the scientific and the technological fields. Since the beginning of the use of numerical methods, the analyst has wondered the accuracy and the reliability of the results that are obtained. Therefore, much effort has been dedicated to estimate the committed error in the simulations. In this paper, we study the a posteriori pointwise error committed in FEM of $2 \mathrm{D}$ transport equations.

An important point in analyzing the error is to know the relationship between the error and the error sources. In the analysis of error estimation, the error sources are identified with the residuals, namely the internal residual inside the elements and the inter-element residuals defined on the element boundaries. Due to the character of the FEM solution, several types of residuals arise. In this paper, the error estimation is decomposed in two components according to the different residuals. A challenge that is tackled is to establish the connection between the different residuals and the error components.

The error estimation is based on the variational multiscale method (VMS), which consists in decomposing the variational form in coarse scales and fine scales. We identify the coarse scales with the FEM solution and fine scales with the error. The VMS theory has been used previously in the error estimation field $[4,19,22,20,21]$. The present error estimate is classified as a residual-based error estimation since the residuals of the coarse scales are employed to estimate the error. There are many works about this type of error estimator. In turn, this error estimation can be divided according in subdomain residual methods and element residual methods. The former estimator employs a patch of elements to estimate the error. For elliptic equations, a general overview can be shown in the papers [38, 15, 43, 37, 39, 34, 42, 33]. On the other hand, the element residual estimators only require the information inside the element and the element boundary to obtain a local error estimate. They usually require to introduce equilibrated residual methods to obtain a well-posed problem. In this field, we can remark the following authors $[2,30,31,7,3,1]$.

Several studies have been made about a posteriori pointwise error previously. Nochetto and Dari et al. [35, 14] developed bounds for the pointwise error in elliptic equations. An important work was made by Prudhomme et al. 
[40] where quantities of interest are employed to determine the pointwise error solving the dual problem in one-dimensional cases. Recently, Irisarri and Hauke [29] developed an a posteriori pointwise error estimation for elliptic problems with application to $2^{\text {nd }}$ and $4^{\text {th }}$-order ODEs making use of fine-scale Green's functions [27]. In previous papers [28], we have analyzed the pointwise error for the $1 \mathrm{D}$ transport equation. Following the same philosophy, the present manuscript extends the methodology to $2 \mathrm{D}$ problems which involves a greater complexity to characterize the error, especially, the error pollution. Bounds for the error pollution, measured in the energy norm, have been analyzed in works of Babuška [6, 8] et al. and Oden et al. [36]. They study the influence of the pollution on local elementwise error estimation making use of Green's functions. If residual-free bubbles and the problem classical Green's function are employed, the technology leads to an exact explicit method for the pointwise error. In practice, however, they can be substituted by bubble functions and free-space Green's functions, in which case, a local projection problem must be solved within each element and a global boundary integral equation, on the boundary domain. As a consequence, this provides a model for the so-called fine-scale Green's functions $[24,26]$ which, basically, are the Green's function that arises from the fine-scale space. The error is analyzed for the heat equation, the reaction-diffusion equation and the convectiondiffusion equation for solutions obtained by the standard Galerkin method and a stabilized method.

The paper is organized as follows: in Section 2, the general variational multiscale framework is analyzed, in which the kinds of residuals are presented. The error estimation formulation is set in Section 3, in which the methodology to estimate the two components of the error is established. In Section 4, numerical examples are shown to corroborate the theoretical formulation. Finally, the last section is devoted to conclusions.

\section{Variational Multiscale formulation}

In this section, the background of the error estimator is presented. The error estimator is based on the VMS theory, in which the test and trial functions are decomposed into fine and coarse scales. This methodology has been extensively applied in the literature to analyze stabilized methods $[24,26]$ and to estimate the error $[18,19,20,21,22,32,34,29,28]$ to estimate the error in elasticity and in fluid mechanics. 
In this paper, we focus on the a posteriori pointwise error estimation for $2 \mathrm{D}$ transport equations. Next, the general methodology to estimate the pointwise error is exposed, and then it is particularized for each differential equation.

Let us express a partial differential equation as

$$
\left\{\begin{array}{rll}
\mathcal{L} u=f & \text { in } & \Omega \\
u=g & \text { on } & \Gamma_{g} \\
\mathcal{B} u=h & \text { on } & \Gamma_{h}
\end{array}\right.
$$

where $\mathcal{L}$ is a generic differential operator. In this work, we consider the following equations:

i. Heat equation: $\mathcal{L} u=-\kappa \Delta u$

ii. Reaction-diffusion equation: $\mathcal{L} u=-\kappa \Delta u+s u$

iii. Convection-diffusion equation: $\mathcal{L} u=-\kappa \Delta u+\boldsymbol{a} \cdot \nabla u$

where $\kappa, s$ and $\boldsymbol{a}$ are the diffusion, the reaction and the convection coefficient, respectively. For the reaction diffusion equation we take $\kappa>0$ and $s>0$. The $\mathcal{B}=\kappa \nabla u \cdot \boldsymbol{n}$ operator arises from integration by parts of the differential operator, $\mathcal{L}$, and it acts on the Neumann boundary; $g$ is the value of the Dirichlet boundary condition and $h$ determines the Neumann boundary condition. Let $\mathcal{S}$ and $\mathcal{V}$ be the standard Sobolev spaces for the trial and the test function, respectively,

$$
\begin{aligned}
& \mathcal{S}=\left\{u \in H^{1}(\Omega) \mid u=g \quad \text { on } \quad \Gamma_{g}\right\} \\
& \mathcal{V}=\left\{v \in H^{1}(\Omega) \mid v=0 \quad \text { on } \quad \Gamma_{g}\right\}
\end{aligned}
$$

The variational formulation of Eq. (1) is: Find $u \in \mathcal{S}$ such that

$$
a(w, u)=(w, f)+(w, h)_{\Gamma_{h}} \quad \forall w \in \mathcal{V}
$$

where $a(\cdot, \cdot)$ is the bilinear form, $(\cdot, \cdot)$ the $L^{2}(\Omega)$ inner product and $(\cdot, \cdot)_{\Gamma_{h}}$ the $L^{2}\left(\Gamma_{h}\right)$ inner product on $\Gamma_{h}$. The FEM consists in meshing the domain, $\Omega$, into $n_{\text {el }}$ non-overlapping elements with domain $\Omega^{e}$. Let $\widetilde{\Omega}$ and $\widetilde{\Gamma}$ denote the union of element interiors, $\Omega^{e}$, and inter-element boundaries, $\Gamma^{e}$, respectively,

$$
\widetilde{\Omega}=\bigcup_{e=1}^{n_{\mathrm{el}}} \Omega^{e} \quad \widetilde{\Gamma}=\bigcup_{e=1}^{n_{\mathrm{el}}} \Gamma^{e} \backslash \Gamma
$$


We define the finite spaces $\mathcal{S}^{h} \subset \mathcal{S}$ and $\mathcal{V}^{h} \subset \mathcal{V}$ for the trial FEM solution and weighting functions, respectively,

$$
\begin{aligned}
& \mathcal{S}^{h}=\left\{u_{h} \in H^{1}(\Omega) \mid u_{h \mid \Omega^{e}} \in \mathbb{P}_{k}, u_{h \mid \Gamma_{g}}=g, \forall \Omega^{e} \in \widetilde{\Omega}\right\} \\
& \mathcal{V}^{h}=\left\{w_{h} \in H^{1}(\Omega) \mid w_{h \mid \Omega^{e}} \in \mathbb{P}_{k}, w_{h \mid \Gamma_{g}}=0, \forall \Omega^{e} \in \widetilde{\Omega}\right\}
\end{aligned}
$$

where $\mathbb{P}_{k}$ denotes the space of polynomials of degree $k$. Thus, the standard Galerkin method reads:

Find $u_{h} \in \mathcal{S}^{h}$ such that

$$
a\left(w_{h}, u_{h}\right)=\left(w_{h}, f\right)+\left(w_{h}, h\right)_{\Gamma_{h}} \quad \forall w_{h} \in \mathcal{V}^{h}
$$

For the convection-diffusion problem, the numerical solution can be unstable when convection is dominant. A typical method to stabilize the FEM solution is the streamline upwind Petrov-Galerkin (SUPG) method [13]. It consists in introducing a stabilizing term in the Galerkin formulation. Accordingly, the SUPG method reads: Find $u_{h} \in \mathcal{S}^{h}$ such that

$$
a_{\mathrm{SUPG}}\left(w_{h}, u_{h}\right)=a\left(w_{h}, u_{h}\right)+a_{\tau}\left(w_{h} ; u_{h}, f\right)=\left(w_{h}, f\right)+\left(w_{h}, h\right)_{\Gamma_{h}} \forall w_{h} \in \mathcal{V}^{h}
$$

The stabilizing term $a_{\tau}(\cdot ; \cdot, \cdot)$ is

$$
a_{\tau}\left(w_{h} ; u_{h}, f\right)=\sum_{\Omega^{e} \in \widetilde{\Omega}} a_{\tau}^{e}\left(w_{h} ; u_{h}, f\right)
$$

where $a_{\tau}^{e}\left(w_{h} ; u_{h}, f\right)=\left(\boldsymbol{a} \cdot \nabla w_{h}, \tau^{e}\left(\mathcal{L} u_{h}-f\right)\right)_{\Omega^{e}}$ and $\tau^{e}=\min \left(\frac{h}{2|\boldsymbol{a}|}, \frac{h^{2}}{12 \kappa}\right)$. The value $h$ is a measure of the element length.

Remark 1. It is worth noting that the origin of stabilized methods lays on the VMS. Taking the standard Galerkin formulation, the stabilized term, $a_{\tau}(\cdot ; \cdot, \cdot)$, represents the effect of the fine scales on the coarse scales. However, in this paper the aim is to estimate the numerical error, irrespective of the employed method. Therefore, both the standard Galerkin and the SUPG solutions are considered coarse scales in our analysis.

Next, the VMS theory is introduced in order to study the error. Basically, it consists in decomposing the solution and test functions into resolved and unresolved scales,

$$
\begin{aligned}
& u=\bar{u}+u^{\prime} \quad \bar{u} \in \overline{\mathcal{S}}, \quad u^{\prime} \in \mathcal{S}^{\prime} \\
& w=\bar{w}+w^{\prime} \quad \bar{w} \in \overline{\mathcal{V}}, \quad w^{\prime} \in \mathcal{V}^{\prime}
\end{aligned}
$$


In our VMS analysis, we identify the coarse scales with the finite element solution and the fine scales with the error, and thereby, $\bar{u}$ is identified with the numerical solution, obtained by Galerkin or SUPG methods. Therefore, the coarse scales for the test functions are $\overline{\mathcal{S}} \equiv \mathcal{S}^{h}$, and for the test functions, $\overline{\mathcal{V}} \equiv \mathcal{V}^{h}$. On the other hand, $\mathcal{S}^{\prime}$ and $\mathcal{V}^{\prime}$ are infinite dimensional spaces. They are defined such that $\mathcal{S}=\overline{\mathcal{S}} \oplus \mathcal{S}^{\prime}$ and $\mathcal{V}=\overline{\mathcal{V}} \oplus \mathcal{V}^{\prime}$

Because of bilinearity, the variational form can be split into coarse scales and fine scales

$$
\begin{aligned}
a(\bar{w}, \bar{u})+a\left(\bar{w}, u^{\prime}\right) & =(\bar{w}, f)+(\bar{w}, h)_{\Gamma_{h}} \quad \forall \bar{w} \in \overline{\mathcal{V}} \\
a\left(w^{\prime}, \bar{u}\right)+a\left(w^{\prime}, u^{\prime}\right) & =\left(w^{\prime}, f\right)+\left(w^{\prime}, h\right)_{\Gamma_{h}} \quad \forall w^{\prime} \in \mathcal{V}^{\prime}
\end{aligned}
$$

Eq. (10a) refers to the coarse scales whereas Eq. (10b) applies to the fine scales. Thus, in order to study the fine scales, we focus on Eq. (10b). This equation can be stated as

$$
a\left(w^{\prime}, u^{\prime}\right)=-\left(w^{\prime}, \mathcal{L} \bar{u}-f\right)_{\widetilde{\Omega}}-\left(w^{\prime}, \llbracket \mathcal{B} \bar{u} \rrbracket\right)_{\widetilde{\Gamma}}-\left(w^{\prime}, \mathcal{B} \bar{u}-h\right)_{\Gamma_{h}}
$$

The jump term, $\llbracket \cdot \rrbracket$, represents the difference of the fluxes on both sides of the element boundaries. In this paper, see Fig. 1, the jump term is defined as in $[25]$

$$
\llbracket q \cdot n \rrbracket=q^{+} \cdot n^{+}+q^{-} \cdot n^{-}
$$

It is worth noting that the terms on the RHS of Eq. (11) represent the three error sources: the internal residuals, the inter-element residuals and the Neumann boundary condition residual.

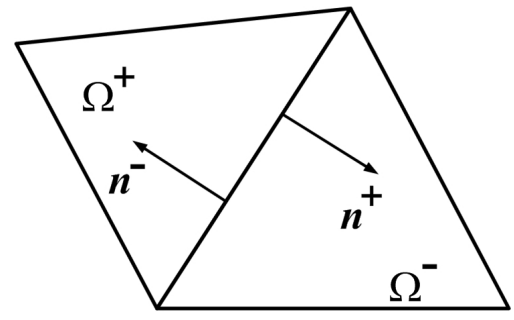

Figure 1: Notation to define the jump across element interfaces.

Remark 2. To analyze the error, the VMS theory based on the Galerkin variational form is employed regardless of the numerical method to compute $\bar{u}$. 


\section{A posteriori error estimation analysis}

The strategy that is followed to estimate the error consists of decomposing the error in two components according to the nature of the residuals that appear in Eq. (11):

$$
u^{\prime}(x)=u_{\text {bub }}^{\prime}(x)+u_{\text {poll }}^{\prime}(x)
$$

The two terms of the decomposition are as follows.

- The internal residual error, $u_{\text {bub }}^{\prime}$, is related to the local internal residual inside the elements, $\mathcal{L} \bar{u}-f$. Thus, it has a local character. This error component is modeled by bubble functions defined within the elements.

- The inter-element error, $u_{\text {poll }}^{\prime}$, represents the pollution error, i.e, the error contribution that appears in an element whose error source is outside the element. This error term arises from three terms:

i. Jumps of the solution on the element boundaries, $\llbracket \mathcal{B} \bar{u} \rrbracket$

ii. Jumps of the local internal residual error, $\llbracket \mathcal{B} u_{\text {bub }}^{\prime} \rrbracket$

iii. The difference between $\mathcal{B} \bar{u}$ and the prescribed value on the Neumann boundary, $h$.

Both components are described in detail in the following subsections. The two-step error estimation has been made beforehand in some works. Huerta and Díez $[15,23]$ solve a first error component on each element making an $h$ discretization on a homogeneous Dirichlet elemental problem instead of a $p$ discretization that we have carried out. Then, the flux jump is taken into account solving local problems that contain the element boundaries using an $h$ discretization again. In [23] the pollution error is estimated projecting over the FEM space.

\subsection{Local internal residual error, $u_{\text {bub }}^{\prime}$}

The error component $u_{\text {bub }}^{\prime}$ is generated by the internal residual $\mathcal{L} \bar{u}-f$ inside each element. The link of the residual with the error can be carried out following the work of Hughes and Sangalli [27], using fine-scale Green's functions $[29,28,22,18]$.

Appendix A explains in more detail how to determine $u_{\text {bub }}^{\prime}$. With this methodology, the error term $u_{\text {bub }}^{\prime}$ can be obtained exactly through the residualfree bubble function concept. However, due to the high computational cost 
to obtain the residual-free bubbles, we have decided to model $u_{\text {bub }}^{\prime}$ by means of bubble functions instead of residual-free bubble functions.

Therefore, the second method to estimate $u_{\text {bub }}^{\prime}$, which is employed in this paper, uses classic bubble functions in combination with the fine-scale variational form (11) that stems from the multiscale decomposition. The strategy that is followed is to subdivide the problem in each element considering that the internal residual error is zero on the boundary elements. Thus, the appropriate space to look for the internal residual error is

$$
\mathcal{S}_{\text {bub }}^{\prime}=\left\{w^{\prime} \in H^{1}(\Omega) \mid w^{\prime}=0 \text { on } \partial \Omega^{e}\right\}
$$

Recalling the fine-scale variational form (11), the internal residual problem is set as: Find $u_{\text {bub }}^{\prime} \in \mathcal{S}_{\text {bub }}^{\prime}$ such that

$$
a\left(w_{\text {bub }}^{\prime}, u_{\text {bub }}^{\prime}\right)=\left(w_{\text {bub }}^{\prime}, f-\mathcal{L} \bar{u}\right) \quad \forall w_{\text {bub }}^{\prime} \in \mathcal{S}_{\text {bub }}^{\prime}
$$

Due to the definition of $\mathcal{S}_{\text {bub }}^{\prime}$, the terms in Eq. (11) belonging to the element boundaries vanish. Eq. (15) must be discretized selecting a finite subspace of $\mathcal{S}_{\text {bub }}^{h} \subset \mathcal{S}_{\text {bub }}^{\prime}$. The way of obtaining a numerical solution of $u_{\text {bub }}^{\prime}$ is solving the following discrete problem:

Find $u_{\text {bub }}^{\prime} \in \mathcal{S}_{\text {bub }}^{h}$ such that

$$
a\left(w_{\text {bub }}^{\prime}, u_{\text {bub }}^{\prime}\right)=\left(w_{\text {bub }}^{\prime}, f-\mathcal{L} \bar{u}\right) \quad \forall w_{\text {bub }}^{\prime} \in \mathcal{S}_{\text {bub }}^{h}
$$

Both the trial functions and the test functions live in the same subspace $\mathcal{S}_{\text {bub }}^{h}$, composed of polynomial bubble functions. Therefore, the internal residual error is established as a linear combination of these bubble functions $b_{i}(\boldsymbol{x})$,

$$
u_{\text {bub }}^{\prime}(\boldsymbol{x})=\sum_{i=1}^{n_{\text {bub }}} c_{i}^{b} b_{i}(\boldsymbol{x})
$$

with $n_{\text {bub }}$ being the number of bubble functions which are chosen and $c_{i}^{b}$ unknown constants to be determined.

The selected bubble functions are defined in the reference element for quadrilaterals and triangles. The first bubble function, $b_{1}(\boldsymbol{x})$, is the simplest polynomial that fulfills to be zero on the element boundary. The successive bubbles are built adding the monomials of the Pascal triangle with center in the barycenter of the element, $c_{e}=\left(\xi_{e}, \eta_{e}\right)$. 
For quadrilaterals: The reference element is: $\Omega_{\text {ref }}=\{(\xi, \eta):-1 \leq \xi \leq$ $1:-1 \leq \eta \leq 1\}$ and $c_{e}=(0,0)$

$$
\begin{aligned}
& b_{1}(\xi, \eta)=\left(1-\xi^{2}\right)\left(1-\eta^{2}\right) \\
& b_{2}(\xi, \eta)=\left(1-\xi^{2}\right) \xi\left(1-\eta^{2}\right) \\
& b_{3}(\xi, \eta)=\left(1-\xi^{2}\right) \eta\left(1-\eta^{2}\right) \\
& b_{4}(\xi, \eta)=\left(1-\xi^{2}\right) \xi \cdot \eta\left(1-\eta^{2}\right) \\
& b_{5}(\xi, \eta)=\left(1-\xi^{2}\right) \xi^{2}\left(1-\eta^{2}\right) \\
& b_{6}(\xi, \eta)=\left(1-\xi^{2}\right) \eta^{2}\left(1-\eta^{2}\right)
\end{aligned}
$$

For triangles: The reference element is: $\Omega_{\mathrm{ref}}=\{(\xi, \eta): 0 \leq \xi \leq 1 ; 0 \leq$ $\eta \leq 1-\xi\}$ and $c_{e}=\left(\xi_{b}, \eta_{b}\right)=(1 / 3,1 / 3)$

$$
\begin{aligned}
& b_{1}(\xi, \eta)=27 \xi \eta(1-\xi-\eta) \\
& b_{2}(\xi, \eta)=27 \xi \eta(1-\xi-\eta)\left(\xi-\xi_{b}\right) \\
& b_{3}(\xi, \eta)=27 \xi \eta(1-\xi-\eta)\left(\eta-\eta_{b}\right) \\
& b_{4}(\xi, \eta)=27 \xi \eta(1-\xi-\eta)\left(\eta-\eta_{b}\right)\left(\xi-\xi_{b}\right) \\
& b_{5}(\xi, \eta)=27 \xi \eta(1-\xi-\eta)\left(\xi-\xi_{b}\right)^{2} \\
& b_{6}(\xi, \eta)=27 \xi \eta(1-\xi-\eta)\left(\eta-\eta_{b}\right)^{2}
\end{aligned}
$$

\subsection{The inter-element error, $u_{\text {poll }}^{\prime}$}

The error term $u_{\text {poll }}^{\prime}$ takes into account the error that is produced by the lack of continuity on the element boundaries. Since the problem is linear, we can split the differential equation as

$$
\mathcal{L} u=\mathcal{L} u^{\prime}+\mathcal{L} \bar{u}=f \quad \text { in } \widetilde{\Omega}
$$

Therefore, if the error is decomposed as $u^{\prime}=u_{\mathrm{bub}}^{\prime}+u_{\text {poll }}^{\prime}$,

$$
\mathcal{L} u_{\text {poll }}^{\prime}=f-\mathcal{L} \bar{u}-\mathcal{L} u_{\text {bub }}^{\prime} \quad \text { in } \widetilde{\Omega}
$$

In case that $u_{\text {bub }}^{\prime}$ is calculated using residual-free bubbles, [11, 12], then $\mathcal{L} u_{\text {bub }}^{\prime}=f-\mathcal{L} \bar{u}$ on $\widetilde{\Omega}$, which implies that $\mathcal{L} u_{\text {poll }}^{\prime}=0$ on $\widetilde{\Omega}$. On the other hand, if $u_{\text {bub }}^{\prime}$ is approximated by bubble functions, then $\mathcal{L} u_{\text {poll }}^{\prime} \approx 0$ on $\widetilde{\Omega}$. 
As a consequence, the error pollution mainly stems from the inter-element jumps. Thus, the inter-element error problem can be set as

$$
\left\{\begin{array}{lll}
\mathcal{L} u_{\text {poll }}^{\prime} & =0 & \text { in } \Omega \backslash \widetilde{\Gamma} \\
\mathcal{L} u_{\text {poll }}^{\prime} & =-\left(\llbracket \mathcal{B} \bar{u} \rrbracket+\llbracket \mathcal{B} u_{\text {bub }}^{\prime} \rrbracket\right) \delta_{\widetilde{\Gamma}} & \text { on } \widetilde{\Gamma} \\
u_{\text {poll }}^{\prime} & =0 & \text { on } \Gamma_{g} \\
\mathcal{B} u_{\text {poll }}^{\prime}=h-\mathcal{B} \bar{u}-\mathcal{B} u_{\text {bub }}^{\prime} & \text { on } \Gamma_{h}
\end{array}\right.
$$

As in the $1 \mathrm{D}$ case [28], the inter-element error can be calculated as a function of free-space Green's functions, $g^{F}\left(\boldsymbol{x}, \boldsymbol{x}_{i}\right)$, or fundamental solutions. We now multiply the two first Eqs. of (22) by the free-space Green's function, $g^{F}$, and integrate over the domain, $\Omega$,

$$
\left\{\begin{array}{l}
\int_{\Omega} \mathcal{L} u_{\text {poll }}^{\prime} g^{F}(\boldsymbol{x}, \boldsymbol{y}) \mathrm{d} \Omega_{y}=0 \quad \text { in } \quad \Omega \backslash \widetilde{\Gamma} \\
\int_{\Omega} \mathcal{L} u_{\text {poll }}^{\prime} g^{F}(\boldsymbol{x}, \boldsymbol{y}) \mathrm{d} \Omega_{y}=-\int_{\widetilde{\Gamma}} g^{F}(\boldsymbol{x}, \boldsymbol{y})\left(\llbracket \mathcal{B} \bar{u} \rrbracket+\llbracket \mathcal{B} u_{\text {bub }}^{\prime} \rrbracket\right) \mathrm{d} \Gamma_{y} \quad \text { on } \quad \widetilde{\Gamma}
\end{array}\right.
$$

Integrating by parts twice the LHS of Eqs. (23) such that,

$$
\begin{aligned}
\int_{\Omega} \mathcal{L} u_{\text {poll }}^{\prime}(\boldsymbol{y}) g^{F}(\boldsymbol{x}, \boldsymbol{y}) \mathrm{d} \Omega_{y}= & \int_{\Omega} u_{\mathrm{poll}}^{\prime}(\boldsymbol{y}) \mathcal{L}^{*} g^{F}(\boldsymbol{x}, \boldsymbol{y}) \mathrm{d} \Omega_{y} \\
& +\int_{\partial \Omega} u_{\text {poll }}^{\prime}(\boldsymbol{y}) \mathcal{B}_{y} g^{F}(\boldsymbol{x}, \boldsymbol{y}) \mathrm{d} \Gamma_{y} \\
& -\int_{\partial \Omega} g^{F}(\boldsymbol{x}, \boldsymbol{y}) \mathcal{B}_{y} u_{\mathrm{poll}}^{\prime}(\boldsymbol{y}) \mathrm{d} \Gamma_{y}
\end{aligned}
$$

where $\mathcal{L}^{*}$ is the adjoint differential operator. Taking advantage that $\mathcal{L}^{*} g^{F}(\boldsymbol{x}, \boldsymbol{y})=$ $\delta(\boldsymbol{x}, \boldsymbol{y})$, the first integral of the RHS of Eq.(24) becomes

$$
\int_{\Omega} u_{\text {poll }}^{\prime}(\boldsymbol{y}) \mathcal{L}^{*} g^{F}(\boldsymbol{x}, \boldsymbol{y}) \mathrm{d} \Omega_{y}=u_{\text {poll }}^{\prime}(\boldsymbol{x})
$$

Then,

$$
\begin{aligned}
\int_{\Omega} \mathcal{L} u_{\text {poll }}^{\prime} g^{F}(\boldsymbol{x}, \boldsymbol{y}) \mathrm{d} \Omega_{y}= & u_{\text {poll }}^{\prime}(\boldsymbol{x})+\int_{\partial \Omega} u_{\text {poll }}^{\prime}(\boldsymbol{y}) \mathcal{B}_{y} g^{F}(\boldsymbol{x}, \boldsymbol{y}) \mathrm{d} \Gamma_{y} \\
& -\int_{\partial \Omega} g^{F}(\boldsymbol{x}, \boldsymbol{y}) \mathcal{B}_{y} u_{\text {poll }}^{\prime}(\boldsymbol{y}) \mathrm{d} \Gamma_{y}
\end{aligned}
$$


Therefore, Eq. (23) becomes

$$
\begin{aligned}
u_{\text {poll }}^{\prime}(\boldsymbol{x})= & -\int_{\widetilde{\Gamma}} g^{F}(\boldsymbol{x}, \boldsymbol{y})\left(\llbracket \mathcal{B} \bar{u} \rrbracket(\boldsymbol{y})+\llbracket \mathcal{B} u_{\text {bub }}^{\prime} \rrbracket(\boldsymbol{y})\right) \mathrm{d} \Gamma_{y}+ \\
& \int_{\partial \Omega} g^{F}(\boldsymbol{x}, \boldsymbol{y}) \mathcal{B}_{y} u_{\text {poll }}^{\prime}(\boldsymbol{y}) \mathrm{d} \Gamma_{y}-\int_{\partial \Omega} u_{\text {poll }}^{\prime}(\boldsymbol{y}) \mathcal{B}_{y} g^{F}(\boldsymbol{x}, \boldsymbol{y}) \mathrm{d} \Gamma_{y} \\
& \text { for all } \boldsymbol{x} \in \Omega
\end{aligned}
$$

The first addend of the RHS in Eq. (27) takes into consideration the error due to the jumps of the fluxes of the FEM solution and $u_{\text {bub }}^{\prime}$ on the element boundaries. The second and the third addends contain information of $u_{\text {poll }}^{\prime}$ and its derivatives on the boundaries.

The representation formula (27) gives the pollution error in the interior of the domain $\Omega$. In case we evaluate this expression on the boundary, a boundary integral equation (BIE) arises, which is set as:

$$
\begin{aligned}
c u_{\text {poll }}^{\prime}\left(\boldsymbol{x}_{0}\right)= & -\int_{\widetilde{\Gamma}} g^{F}\left(\boldsymbol{x}_{0}, \boldsymbol{y}\right)\left(\llbracket \mathcal{B} \bar{u} \rrbracket(\boldsymbol{y})+\llbracket \mathcal{B} u_{\text {bub }}^{\prime} \rrbracket(\boldsymbol{y})\right) \mathrm{d} \Gamma_{y}+ \\
& \int_{\partial \Omega} g^{F}\left(\boldsymbol{x}_{0}, \boldsymbol{y}\right) \mathcal{B}_{y} u_{\text {poll }}^{\prime}(\boldsymbol{y}) \mathrm{d} \Gamma_{y}-\int_{\partial \Omega} u_{\text {poll }}^{\prime}(\boldsymbol{y}) \mathcal{B}_{y} g^{F}\left(\boldsymbol{x}_{0}, \boldsymbol{y}\right) \mathrm{d} \Gamma_{y}
\end{aligned}
$$

The constant $c$ takes different values according to the smoothness of the boundary where $\boldsymbol{x}_{0}$ is sited. If the boundary is smooth, $c=\frac{1}{2}$ (see BEM bibliography, for instance $[9,10])$. The value of $c$ for a corner is $c=\frac{\theta}{2 \pi}$, where $\theta$ is the internal angle of the corner in radians. The unknowns in Eq. (28) are $\mathcal{B}_{y} u_{\text {poll }}^{\prime}$ on $\Gamma_{g}$ and $u_{\text {poll }}^{\prime}$ on $\Gamma_{h}$.

The boundary integral equation, expressed in Eq. (28), is solved applying Boundary Element Method (BEM) concepts [9, 10]. There are several methodologies to tackle the BEM. We consider two methodologies according to the problem boundary conditions.

Remark 3. Eq. (28) could have been expressed as a function of the classic Green's function. In this case, this equation would give explicitly $u_{\text {poll }}^{\prime}(\boldsymbol{x})$. However, due to the difficulty to obtain the classic Green's function for each problem and domain, we present a method based on free-space Green's functions, which requires solving a small boundary problem. 


\subsubsection{Treatment of problems with Dirichlet boundary conditions}

The way of treating the contour integral in Eq. (28) on Dirichlet boundary conditions is simplified by the fact that we know that $u_{\text {poll }}^{\prime}=0$ on the whole Dirichlet boundary,

$$
\begin{aligned}
0= & -\int_{\widetilde{\Gamma}} g^{F}\left(\boldsymbol{x}_{0}, \boldsymbol{y}\right)\left(\llbracket \mathcal{B} \bar{u} \rrbracket(\boldsymbol{y})+\llbracket \mathcal{B} u_{\text {bub }}^{\prime} \rrbracket(\boldsymbol{y})\right) \mathrm{d} \Gamma_{y}+ \\
& \int_{\partial \Omega} g^{F}\left(\boldsymbol{x}_{0}, \boldsymbol{y}\right) \mathcal{B}_{y} u_{\text {poll }}^{\prime}(\boldsymbol{y}) \mathrm{d} \Gamma_{y} \text { for all } \quad \boldsymbol{x}_{0} \in \partial \Omega
\end{aligned}
$$

The only unknown in Eq. (29) is the derivative of $u_{\text {poll }}^{\prime}, \mathcal{B}_{y} u_{\text {poll }}^{\prime}$, on the boundary which plays the role of a density function $\chi(\boldsymbol{y})$ that has to satisfy the homogeneous Dirichlet boundary condition,

$$
\int_{\partial \Omega} g^{F}\left(\boldsymbol{x}_{0}, \boldsymbol{y}\right) \chi(\boldsymbol{y}) \mathrm{d} \Gamma_{y}=\int_{\widetilde{\Gamma}} g^{F}\left(\boldsymbol{x}_{0}, \boldsymbol{y}\right)\left(\llbracket \mathcal{B} \bar{u} \rrbracket(\boldsymbol{y})+\llbracket \mathcal{B} u_{\text {bub }}^{\prime} \rrbracket(\boldsymbol{y})\right) \mathrm{d} \Gamma_{y}
$$

In this problem, the delta-delta collocation method can be employed to enforce Eq. (30) at a set of $N$ points uniformly spaced on the boundary called $\boldsymbol{x}_{b, i}$ for $i=1, \ldots, N$. Thus, for each point $i$ the following equation is imposed,

$$
\sum_{j=1}^{N} g^{F}\left(\boldsymbol{x}_{b, i}, \boldsymbol{x}_{b, j}\right) \chi_{b, j}=\int_{\widetilde{\Gamma}} g^{F}\left(\boldsymbol{x}_{b, i}, \boldsymbol{y}\right)\left(\llbracket \mathcal{B} \bar{u} \rrbracket(\boldsymbol{y})+\llbracket \mathcal{B} u_{\text {bub }}^{\prime} \rrbracket(\boldsymbol{y})\right) \mathrm{d} \Gamma_{y}
$$

where $\chi_{b, j}$ are the unknown weights to be determined.

Finally, once the coefficients $\chi_{b, j}$ are known, $u_{\text {poll }}^{\prime}$ on the domain is obtained as

$$
\begin{aligned}
u_{\text {poll }}^{\prime}(\boldsymbol{x})= & -\int_{\widetilde{\Gamma}} g^{F}(\boldsymbol{x}, \boldsymbol{y})\left(\llbracket \mathcal{B} \bar{u} \rrbracket(\boldsymbol{y})+\llbracket \mathcal{B} u_{\text {bub }}^{\prime} \rrbracket(\boldsymbol{y})\right) \mathrm{d} \Gamma_{y}+ \\
& \sum_{j=1}^{N} g^{F}\left(\boldsymbol{x}, \boldsymbol{x}_{b, j}\right) \chi_{b, j} \text { for all } \boldsymbol{x} \in \Omega
\end{aligned}
$$

3.2.2. Treatment of problems with Neumann and Dirichlet boundary conditions

Compared to problems with only Dirichlet boundary conditions, now the main difference to face is the singular integral that appears with the derivative of the Green's function in the BIE and the steep change in the type 
of boundary conditions from Dirichlet to Neumann. For this reason, the collocation delta-delta method is not convenient to handle the singularities. Thus, a more elaborated method is invoked

Calling $q_{\text {poll }}^{\prime}=\mathcal{B} u_{\text {poll }}^{\prime}$, the inter-element error, $u_{\text {poll }}^{\prime}$ and its flux along the boundary are approximated as

$$
\begin{aligned}
& u_{\text {poll }}^{\prime}(\boldsymbol{x})=\sum_{i=1}^{N} u_{\text {poll,i }}^{\prime} \psi_{i}(\boldsymbol{x}) \text { for } i=1, \ldots, N \\
& q_{\text {poll }}^{\prime}(\boldsymbol{x})=\sum_{i=1}^{N} q_{\text {poll, }}^{\prime} \psi_{i}(\boldsymbol{x}) \text { for } i=1, \ldots, N
\end{aligned}
$$

where $N$ are the number of boundary elements and with $u_{\mathrm{poll}, \mathrm{i}}^{\prime}$ and $q_{\mathrm{poll}, \mathrm{i}}^{\prime}$ being the unknown coefficients and $\psi_{i}(\boldsymbol{x})$ the approximation basis functions. In this work, $\psi_{i}(\boldsymbol{x})$ are considered constants, which simplify and reduce the computational effort. Now, we introduce Eq. (33) and discretize Eq. (28) in $N$ elements along the boundary

$$
\begin{aligned}
c u_{\text {poll, } \mathrm{i}}^{\prime}= & -\int_{\widetilde{\Gamma}} g^{F}\left(\boldsymbol{x}_{0}, \boldsymbol{y}\right)\left(\llbracket \mathcal{B} \bar{u} \rrbracket(\boldsymbol{y})+\llbracket \mathcal{B} u_{\text {bub }}^{\prime} \rrbracket(\boldsymbol{y})\right) \mathrm{d} \Gamma_{y}+ \\
& \sum_{j=1}^{N} q_{\text {poll }, \mathrm{j}}^{\prime}\left(\int_{\partial \Omega} g^{F}\left(\boldsymbol{x}_{0}, \boldsymbol{y}\right) \psi_{j} \mathrm{~d} \Gamma\right)-\sum_{j=1}^{N} u_{\text {poll, }, \mathrm{j}}^{\prime}\left(\int_{\partial \Omega} \mathcal{B}_{y} g^{F}\left(\boldsymbol{x}_{0}, \boldsymbol{y}\right) \psi_{j} \mathrm{~d} \Gamma\right) \\
& \text { for } j=1, \ldots, N
\end{aligned}
$$

where the only unknowns are $u_{\mathrm{poll}, \mathrm{j}}^{\prime}$ on $\Gamma_{h}$ and $q_{\mathrm{poll}, \mathrm{j}}^{\prime}$ on $\Gamma_{g}$. On the Dirichlet boundary, we know that $u_{\text {poll }}^{\prime}=0$ and $q_{\text {poll }}^{\prime}$ is unknown. On the other hand, on the Neumann boundary, the unknown is $u_{\text {poll }}^{\prime}$ and the flux $q_{\text {poll }}^{\prime}$ is

$$
\begin{aligned}
q_{\text {poll }}^{\prime}= & h-\mathcal{B} \bar{u}\left(\boldsymbol{x}_{0}\right)-\mathcal{B} u_{\text {bub }}^{\prime}\left(\boldsymbol{x}_{0}\right) \\
& \text { for } \boldsymbol{x}_{0} \in \Gamma_{h}
\end{aligned}
$$

It is worth noting that at any boundary point there is only one unknown to be determined. Therefore, we form a system of equations evaluating (34) for $i=1, \ldots, N$.

Once the components $u_{\mathrm{poll}, \mathrm{j}}^{\prime}$ and $q_{\mathrm{poll}, \mathrm{j}}^{\prime}$ for $j=1, \ldots, N$ are known, the 
inter-element error can be evaluated at any point $\boldsymbol{x} \in \Omega$ using Eq. (36)

$$
\begin{aligned}
u_{\text {poll }}^{\prime}(\boldsymbol{x})= & -\int_{\widetilde{\Gamma}} g^{F}(\boldsymbol{x}, \boldsymbol{y})\left(\llbracket \mathcal{B} \bar{u} \rrbracket(\boldsymbol{y})+\llbracket \mathcal{B} u_{\text {bub }}^{\prime} \rrbracket(\boldsymbol{y})\right) \mathrm{d} \Gamma_{y}+ \\
& \sum_{j=1}^{N} q_{\text {poll, } \mathrm{j}}^{\prime}\left(\int_{\partial \Omega} g^{F}(\boldsymbol{x}, \boldsymbol{y}) \psi_{j} \mathrm{~d} \Gamma\right)-\sum_{j=1}^{N} u_{\mathrm{poll}, \mathrm{j}}^{\prime}\left(\int_{\partial \Omega} \frac{\partial g^{F}(\boldsymbol{x}, \boldsymbol{y})}{\partial n_{y}} \psi_{j} \mathrm{~d} \Gamma\right) \\
& \text { for } \boldsymbol{x} \in \Omega
\end{aligned}
$$

For the numerical examples, the first integral that appears on the RHS both in Eq. (36) and in Eq. (32) is carried out with a uniform discretization on each boundary element using one-point Gauss quadrature rule. On the other hand, the BEM with constant elements or the collocation method that are employed to solve the BIE provides first order for the measure of the pointwise error. This convergence rate is corroborated in the numerical examples.

Remark 4. The extension to 3D problems does not imply more conceptual complexity since the same steps as in this paper must be followed. In 3D problems, the internal residual error, $u_{\mathrm{bub}}^{\prime}$, is composed of elemental bubbles that are zero on the faces of the elements. On the other hand, the discontinuity of the shape function derivatives must be considered on the faces of the elements instead of on the element edges that we have seen in 2D.

Remark 5. Taking advantage of the pointwise error estimation, adaptive refinement meshes can be made. An example of a mesh refinement process is carried out later.

\section{Numerical results}

In order to validate the proposed error estimator, we present several examples with application to the heat equation, the reaction-diffusion equation and the convection-diffusion equation.

\subsection{Heat equation}

The differential equation for the heat equation is

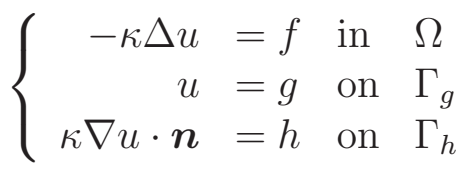


In this case, $\mathcal{B} u=\kappa \nabla u \cdot \boldsymbol{n}$. It is well-known that the free-space Green's function or the fundamental solution is

$$
g^{F}\left(\boldsymbol{x}, \boldsymbol{x}_{i}\right)=-\frac{1}{2 \pi \kappa} \ln \left(\sqrt{\left(\boldsymbol{x}-\boldsymbol{x}_{i}\right)^{2}}\right)
$$

where $\boldsymbol{x}_{i}$ are the coordinates of the unit source point.

\subsubsection{Heat equation with Dirichlet boundary conditions}

A domain $\Omega=(0,1) \times(0,1)$ with homogeneous Dirichlet boundary condition except on the upper edge where a triangular temperature distribution is applied: $0^{\circ} \mathrm{C}$ at $(0,0), 10^{\circ} \mathrm{C}$ at $(0.5,0)$ and $0^{\circ} \mathrm{C}$ at $(1,0)$. The source term is zero, $f=0$. Two kind of meshes are analyzed:

- $4 \times 4$ bilinear quadrilateral elements

- 16 linear triangle elements

We begin with the $4 \times 4$ bilinear quadrilateral elements. The numerical and the reference solution are depicted in Fig. 2. The reference solution is considered close to the exact one and it is obtained using a fine mesh of $100 \times 100$ elements.

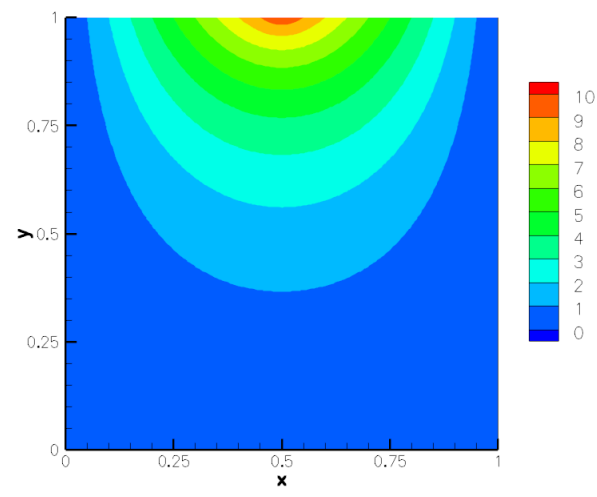

(a) Reference solution

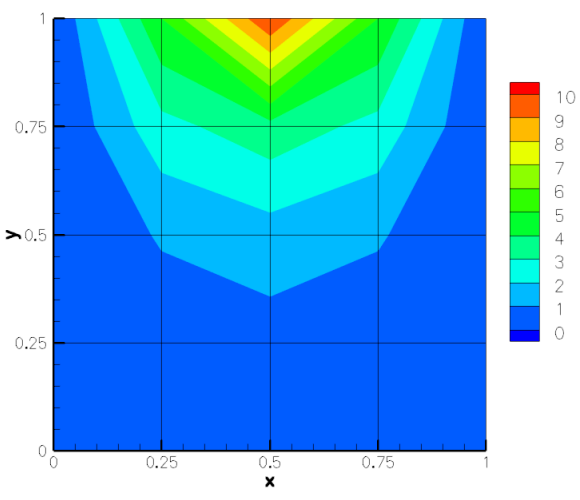

(b) FEM solution

Figure 2: Heat equation. Dirichlet problem. Reference and FEM solution.

This problem has the particular characteristic that the residual $\mathcal{L} \bar{u}-f$ is zero since $f=0$ and $\mathcal{L} \bar{u}=0$. Therefore, the internal residual error is zero 
and the error is uniquely expressed by the inter-element residual error. As we have a problem with Dirichlet boundary conditions on the whole boundary, Eq. (31) is employed discretizing the boundary with 10 constant elements on each edge of the FEM elements located on the boundary. Therefore, in this case, we employed 160 constant BEM elements along the domain boundary. Once the coefficients $\psi_{b, i}$ are determined, the error term $u_{\text {poll }}^{\prime}$ is recovered via Eq. (32).

Figs. 3 and 4 represent the reference error and the predicted error in $2 \mathrm{D}$ and 3D graphics. It can be appreciated that the predicted error and the reference error are very similar.

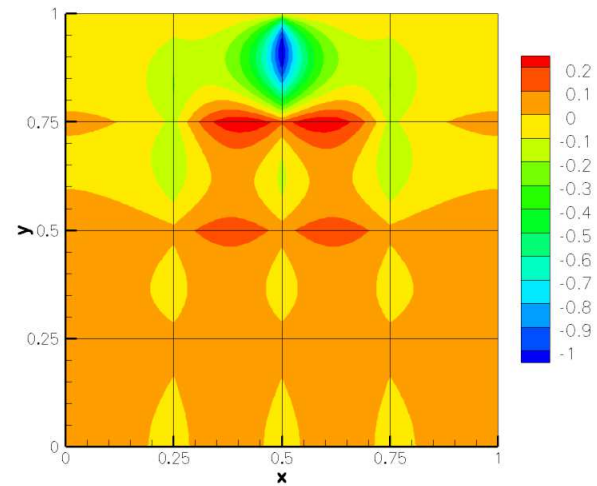

(a) Reference error

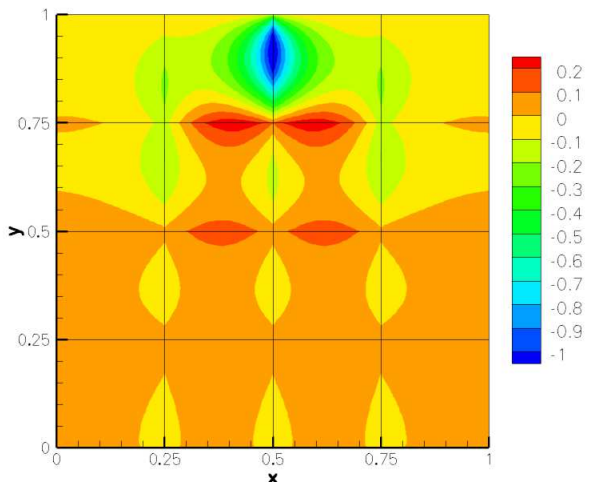

(b) Estimated error

Figure 3: Heat equation. Dirichlet problem. Reference and estimated error.

For triangular elements, the internal residual is again zero, $\mathcal{L} \bar{u}-f=0$. Thus, the pointwise error can be determined by the same methodology as with the bilinear quadrilateral, using Eq. (32).

Fig. 5 depicts the mesh that has been employed for the FEM solution. The reference and the estimated error are shown in Fig. 6 and are practically identical.

In order to check the rate of convergence of the estimated pointwise error, we plot a log-log graphic in Fig. 7, in which the committed error in the computation of the FEM error is analyzed. Specifically, it represents the error at the center of a element as a function of the discretization size, $h$, of the element boundaries and domain boundary. In this example, we take the point $(0.375,0.625)$. We recall that the collocation method is used to solve 


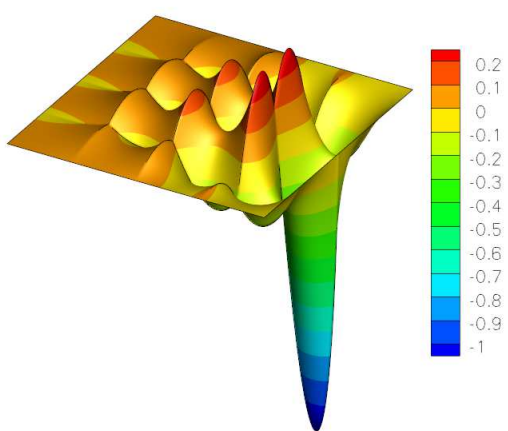

(a) Reference error

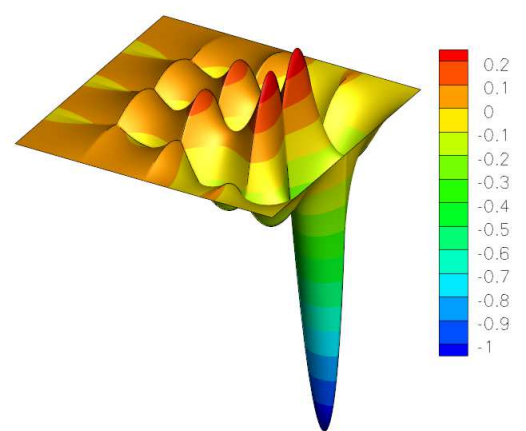

(b) Estimated error

Figure 4: Heat equation. Dirichlet problem. Reference and estimated error in 3D.

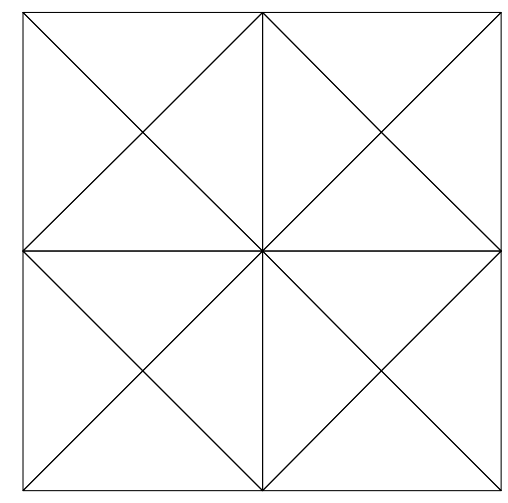

Figure 5: Mesh based on linear triangles.

the BIE and one-point Gauss quadrature is applied to the contour integrals on the element boundaries. It can be observed that first order convergence rates are provided as it is expected according to convergence theory.

\subsubsection{Heat equation with Dirichlet and Neumann boundary conditions}

A domain $\Omega=(0,1) \times(0,1)$ with $f=0$, and homogeneous Dirichlet boundary conditions except on the upper edge where there is a Neumann boundary condition in the interval $(0.25,0.75)$. Fig. 8 depicts the problem with the corresponding boundary conditions.

The strong form of the problem is 


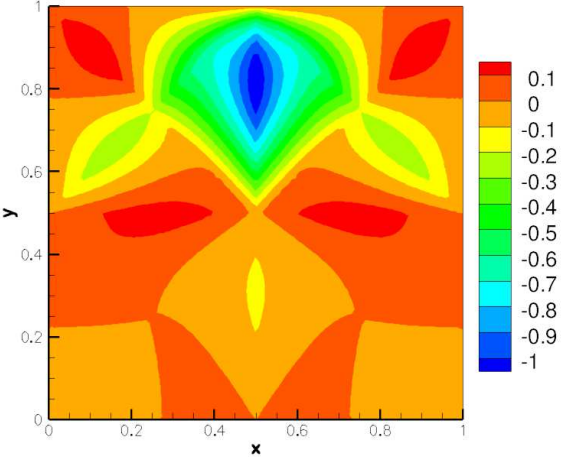

(a) Reference error

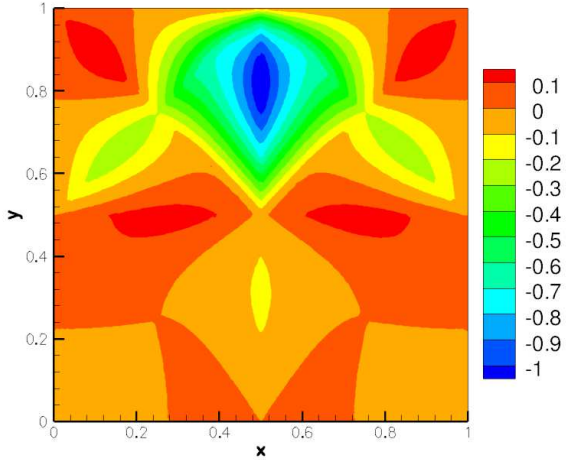

(b) Estimated error

Figure 6: Heat equation. Dirichlet problem and linear triangles. Reference and estimated error.

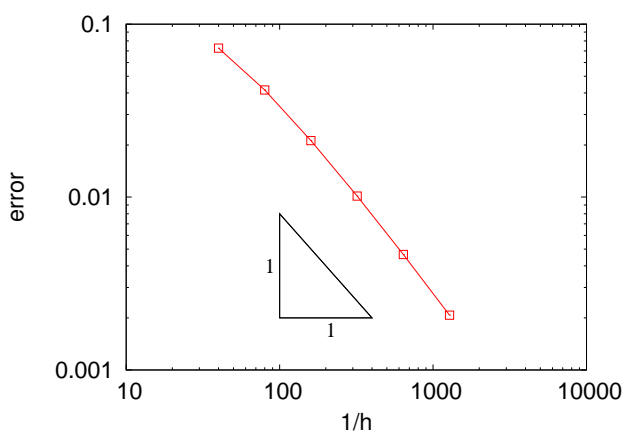

Figure 7: Convergence rate of the pointwise error estimation at $(0.375,0.625)$

$$
\left\{\begin{aligned}
-\kappa \Delta u & =0 & & \text { in } \Omega \\
u & =0 & & \text { on } \Gamma_{g 1} \\
u & =10 & & \text { on } \Gamma_{g 2} \\
u & =10-10 y & & \text { on } \Gamma_{g 3} \\
\kappa \nabla u \cdot \boldsymbol{n} & =0 & & \text { on } \Gamma_{h}
\end{aligned}\right.
$$

where $\Gamma_{g}=\Gamma_{g 1} \bigcup \Gamma_{g 2} \bigcup \Gamma_{g 3}$ is the Dirichlet boundary and $\Gamma_{h}$ is the Neumann 


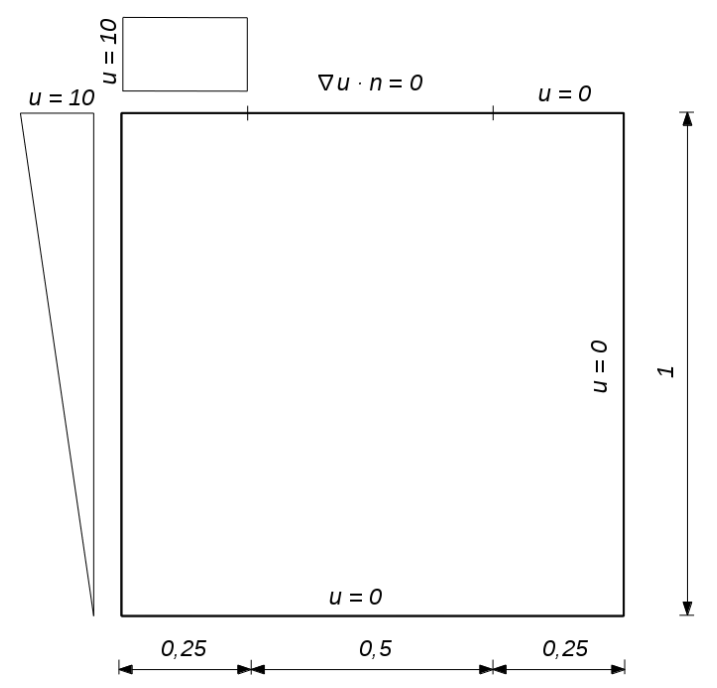

Figure 8: Heat equation. Problem with Neumann and Dirichlet boundary conditions.

boundary. $\Gamma_{h}, \Gamma_{g 1}$ and $\Gamma_{g 2}$ are defined as

$$
\begin{aligned}
& \Gamma_{h}=\{(x, 1) ; 0.25<x<0.75\} \\
& \Gamma_{g 2}=\{(x, 1) ; 0 \leq x \leq 0.25\} \\
& \Gamma_{g 3}=\{(0, y) ; 0 \leq y<1\} \\
& \Gamma_{g 1}=\left\{(x, y) \in \Gamma \backslash\left(\Gamma_{g 1} \bigcup \Gamma_{g 2} \bigcup \Gamma_{h}\right)\right\}
\end{aligned}
$$

The coarse mesh is made of $4 \times 4$ bilinear quadrilateral elements. The numerical solution is depicted in Fig. 9 together with the reference solution.

As in the latter case, there is no internal residual in this problem and the only error component is $u_{\text {poll }}^{\prime}$. The great difference between this problem and the previous one is that now the error is not zero on the whole boundary due to the Neumann boundary condition.

For estimating the pollution error, the BIE is solved discretizing the boundary as is expressed in Eq. (34), The boundary has been discretized with 160 constant BEM elements.

Fig. 10 represents the error estimation in $3 \mathrm{D}$ representation. It can be observed that the error is sharp on the singularities around the Neumann boundary condition, especially, at the point of encounter between Neumann and Dirichlet boundary conditions. Fig. 11 depicts also the predicted error and the reference one. 


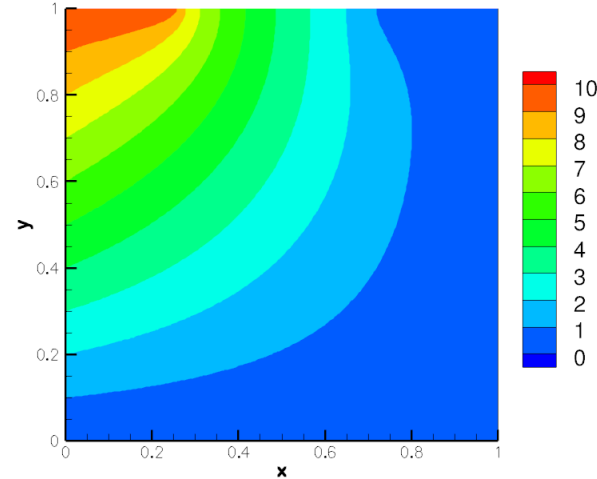

(a) Reference solution

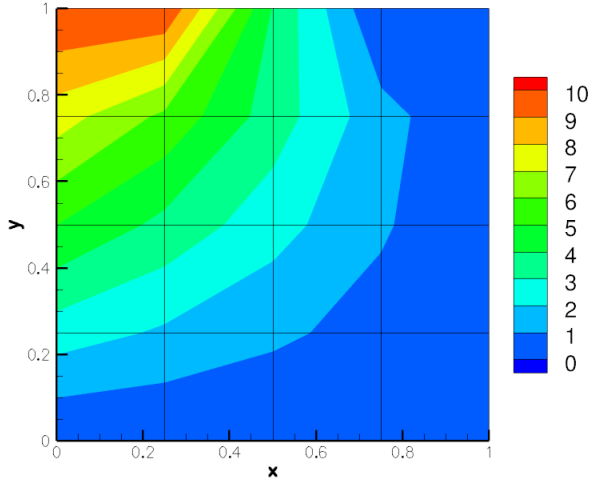

(b) FEM solution

Figure 9: Heat equation. Reference and FEM solution with Neumann and Dirichlet boundary conditions.

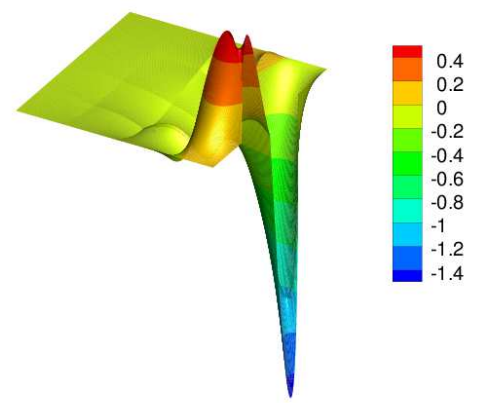

(a) Reference error

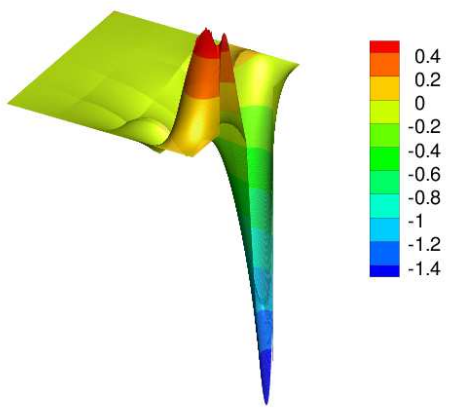

(b) Estimated error

Figure 10: Heat equation. Reference and estimated error with Neumann and Dirichlet boundary conditions in $3 \mathrm{D}$ view.

\subsection{The reaction-diffusion equation}

The reaction-diffusion equation is

$$
\left\{\begin{array}{rlll}
-\kappa \Delta u+s u & =f & \text { in } & \Omega \\
u & =g & \text { on } & \Gamma_{g} \\
\kappa \nabla u \cdot \boldsymbol{n} & =h & \text { on } & \Gamma_{h}
\end{array}\right.
$$




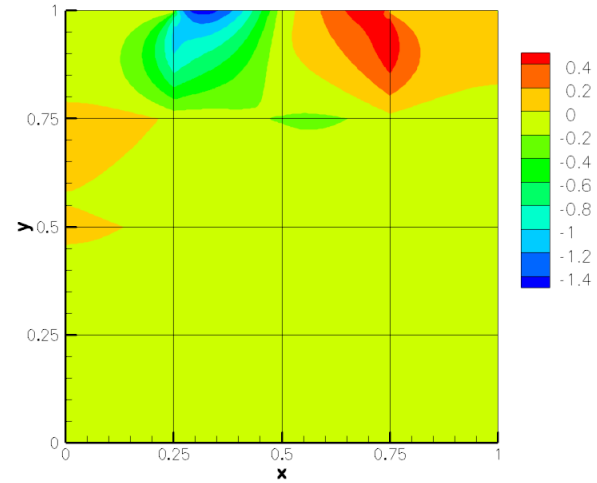

(a) Reference error

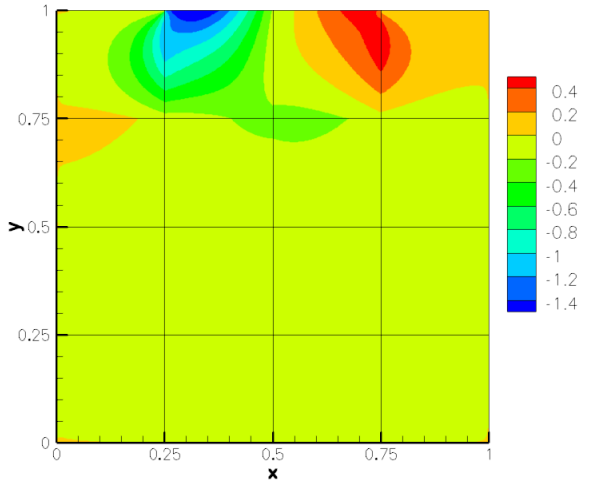

(b) Estimated error

Figure 11: Heat equation. Reference and estimated error with Neumann and Dirichlet boundary conditions.

where $\kappa$ and $s$ are the diffusion and the reaction coefficients, respectively.

The free-space Green's function for the reaction-diffusion equation is defined as [41],

$$
g^{F}(x, y ; \xi, \eta)=\frac{1}{2 \pi \kappa} K_{0}\left(\lambda_{r} r\right)
$$

where $K_{0}$ denotes the modified Bessel function of the second kind, $r^{2}=$ $(\xi-x)^{2}+(\eta-y)^{2}$ and $\lambda_{r}^{2}=s / \kappa$

The proposed reaction-diffusion problem consists of a domain $\Omega=(0,1) \times$ $(0,1)$ and homogeneous Dirichlet boundary condition. The source term is $f=1$ and the coefficients of the equation are $\kappa=1$ and $s=10$. Two kind of meshes are considered:

- $4 \times 4$ bilinear quadrilateral elements

- 16 linear triangle elements

The reference solution has been obtained with a fine mesh made of $100 \times$ 100 elements. Fig. 12 represents both the reference and the FEM solution.

As described in Section 3, the error estimation is decomposed in internal residual error and inter-element error. 


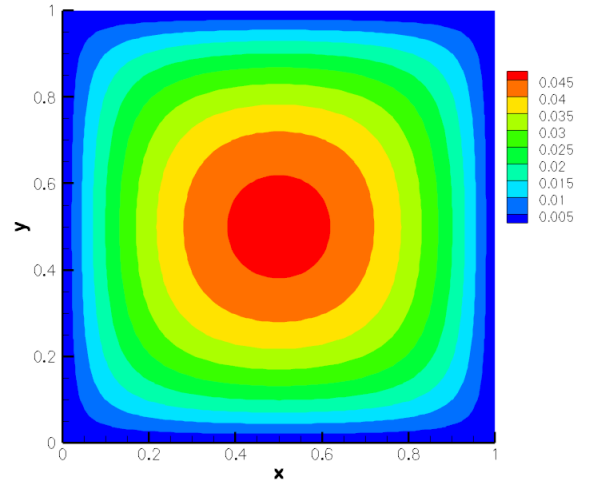

(a) Reference solution

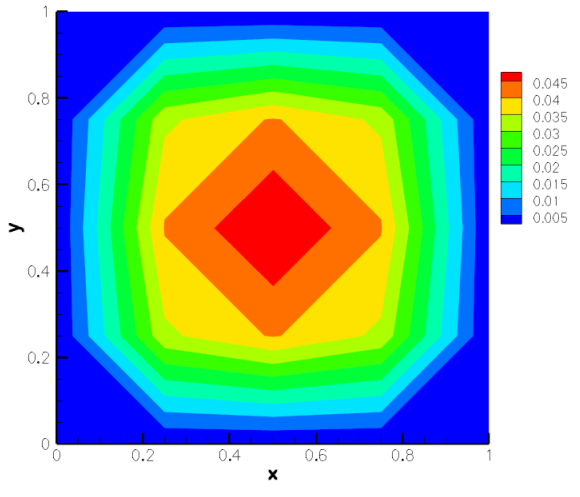

(b) FEM solution

Figure 12: Reaction-diffusion equation. Reference and FEM solution for quadrilaterals.

\subsubsection{Internal residual error, $u_{\mathrm{bub}}^{\prime}$}

The internal residual error comes from the internal residuals and is located inside each element. This error component, $u_{\mathrm{bub}}^{\prime}$, is expressed by a combination of bubble functions that satisfies Eq. (16) in each element.

Fig. 13 represents the internal residual error, $u_{\text {bub }}^{\prime}$, selecting up to the bubble $b_{3}(\boldsymbol{x})$ of Eq. (18) and (19) for quadrilaterals and triangles, respectively. In this case, the internal residual is $s \bar{u}-f$ since bilinear or linear elements are employed, and therefore, $-\kappa \Delta \bar{u}=0$.

\subsubsection{Inter-element error, $u_{\text {poll }}^{\prime}$}

As for the inter-element error, we have to take into account both the jump on the boundary element due to the lack of continuity of the FEM solution and the internal residual error, $u_{\mathrm{bub}}^{\prime}$. The inter-element error, $u_{\text {poll }}^{\prime}$, is depicted Fig. 14 in 2D and 3D views. The BIE is solved discretizing with 160 constant BEM elements placed uniformly on the domain boundary.

Summing the internal residual error, $u_{\text {bub }}^{\prime}$, and the inter-element residual error, $u_{\text {poll }}^{\prime}$, the pointwise error estimation is observed in Fig. 15 where it is compared with the reference one.

For triangle elements, we have selected the mesh represented in Fig. 16a. The FEM solution is depicted in Fig. 16b. The error estimation is obtained following the same steps as with quadrilaterals. Fig. 17 shows the reference and the estimated error. 


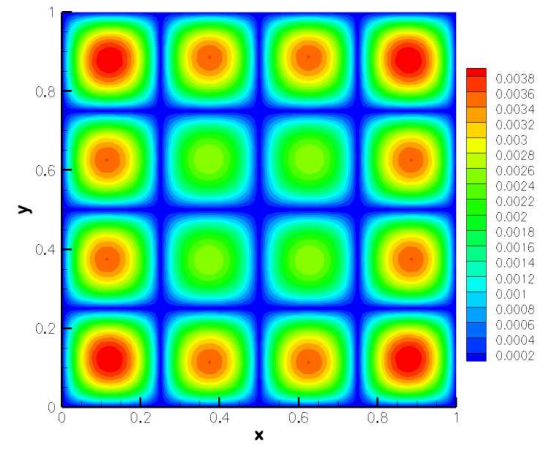

(a) $2 \mathrm{D}$ view

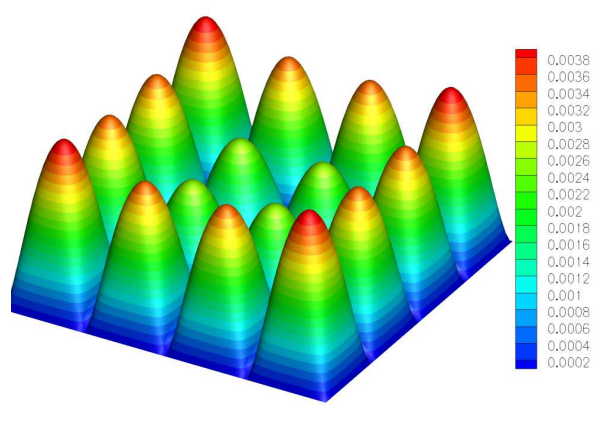

(b) $3 \mathrm{D}$ view

Figure 13: Reaction-diffusion equation. Internal residual error for quadrilaterals.

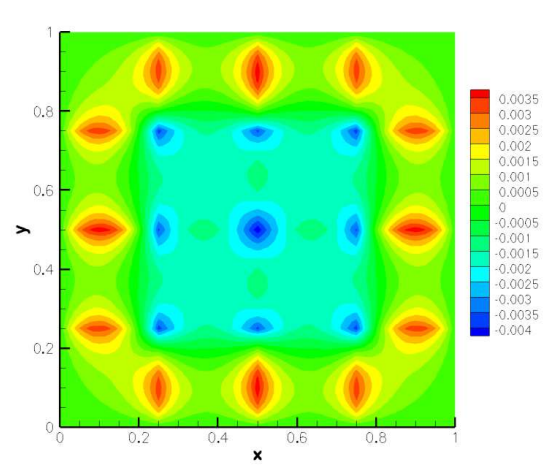

(a) $2 \mathrm{D}$ view

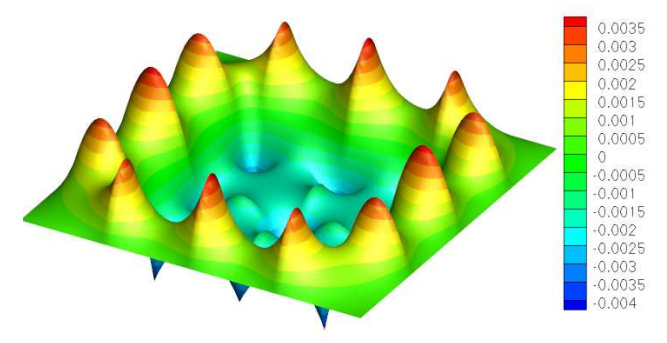

(b) $3 \mathrm{D}$ view

Figure 14: Reaction-diffusion equation. Inter-element error for quadrilaterals.

\subsection{Convection-diffusion equation}

The convection-diffusion problem is expressed as

$$
\left\{\begin{array}{rlll}
-\kappa \Delta u+\boldsymbol{a} \cdot \nabla u & =f & \text { in } & \Omega \\
u & =g & \text { on } & \Gamma_{g} \\
\kappa \nabla u \cdot \boldsymbol{n} & =h & \text { on } & \Gamma_{h}
\end{array}\right.
$$

where $k$ and $\boldsymbol{a}=\left(a_{x}, a_{y}\right)$ are the diffusive and convection coefficients, respec- 


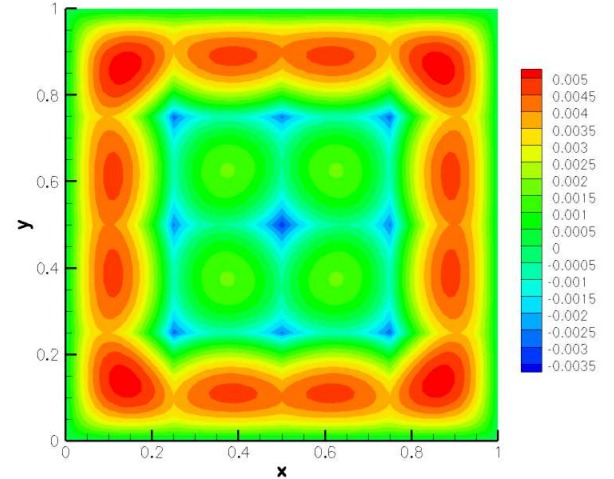

(a) Reference error

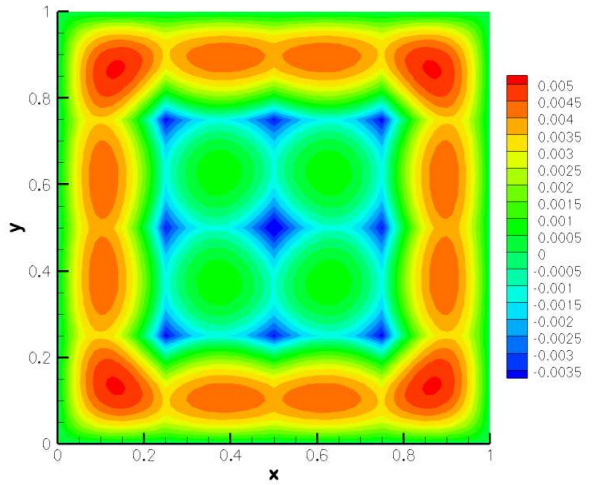

(b) Estimated error

Figure 15: Reaction-diffusion equation. Reference and estimated error for quadrilaterals.

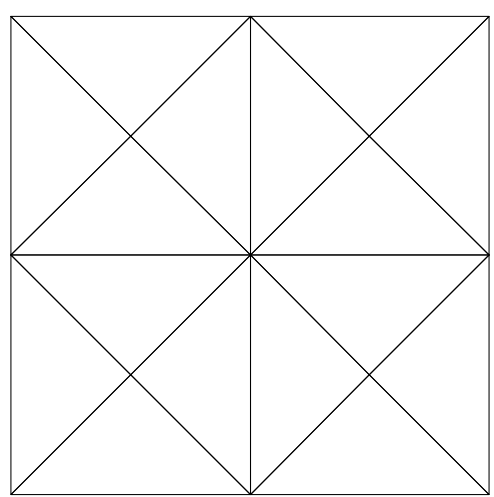

(a) Mesh

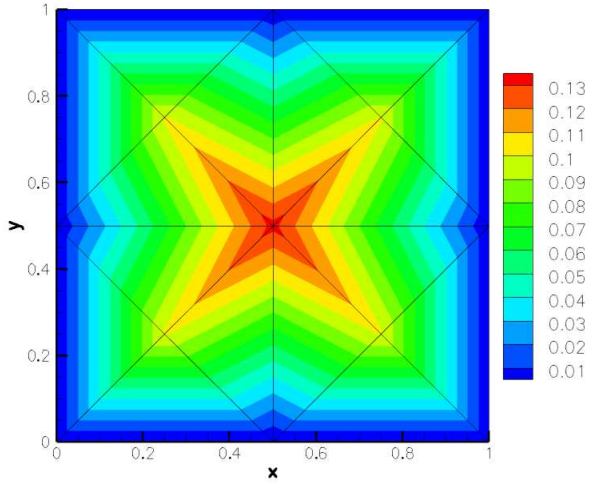

(b) FEM solution

Figure 16: Reaction-diffusion equation. Mesh and numerical solution for triangles.

tively.

The process to estimate the error is the same as has been explained. The internal residual error is estimated via Eq. (15) particularized to convectiondiffusion equation. The free-space Green's functions for convection-diffusion equation is defined as [41],

$$
g^{F}(x, y ; \xi, \eta)=\frac{1}{2 \pi \kappa} \exp \left(\frac{b_{x}(\xi-x)+b_{y}(\eta-y)}{2 \kappa}\right) K_{0}\left(\lambda_{a} r\right)
$$




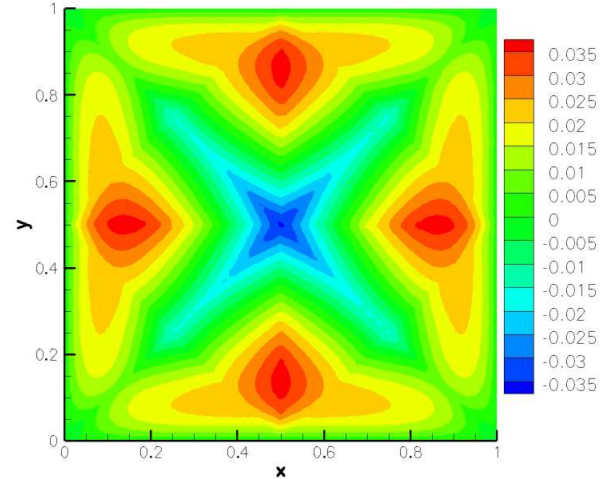

(a) Reference error

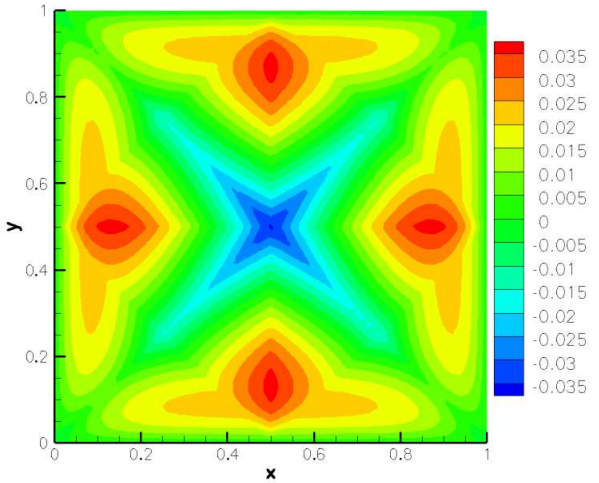

(b) Estimated error

Figure 17: Reaction-diffusion equation. Reference and estimated error for triangles.

where $K_{0}$ denotes the modified Bessel function of the second kind, $r^{2}=$ $(\xi-x)^{2}+(\eta-y)^{2}$ and $\lambda_{a}^{2}=\left(b_{x}^{2}+b_{y}^{2}\right) /(2 \varepsilon)^{2}$.

The problem for the convection-diffusion equation is a domain $\Omega=(0,1) \times$ $(0,1)$ with $\kappa=0.03$ and an oblique advection $\boldsymbol{a}=(1,1)$. The source term is $f=1$. The reference solution has been obtained with a fine mesh made of $100 \times 100$ elements. The error estimation is analyzed both using the standard Galerkin and the SUPG method.

\subsubsection{Error estimation for the standard Galerkin method}

Fig. 18 represents both the reference and the FEM solution for the standard Galerkin using a coarse mesh of $4 \times 4$ bilinear elements. The numerical solution is unstable and spurious oscillations appear along the domain.

For this equation and using bilinear quadrilaterals, the internal residual source is $f-\boldsymbol{a} \cdot \nabla \bar{u}$ obtained by Eq. (16). The error term $u_{\text {bub }}^{\prime}$ is approximated with bubbles up to $b_{6}(\boldsymbol{x})$, solving Eq. (16). It is represented in Fig. 19.

For the inter-element error we have to consider the jump on the element interfaces of the numerical solution and the internal residual error. Also, in order to satisfy the boundary conditions we solve the BIE via the collocation method. Fig. 20 shows the inter-element error. As we have only Dirichlet boundary conditions, the collocation method is applied to solve the BIE. As in the previous examples, 160 constant BEM elements are posed on the domain boundary spaced uniformly. 


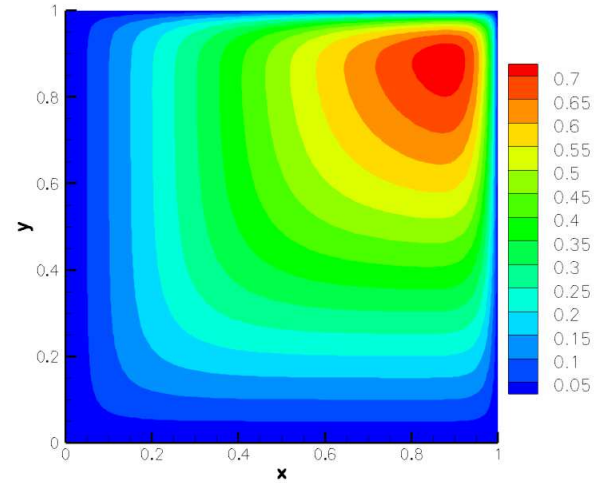

(a) Reference solution

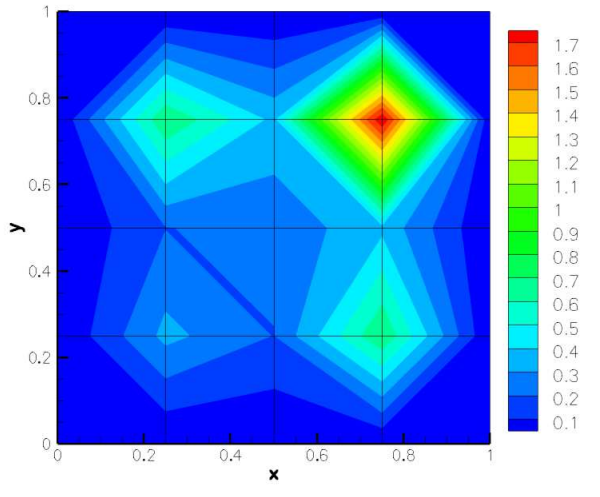

(b) FEM solution

Figure 18: Convection-diffusion equation. Reference and FEM solution.

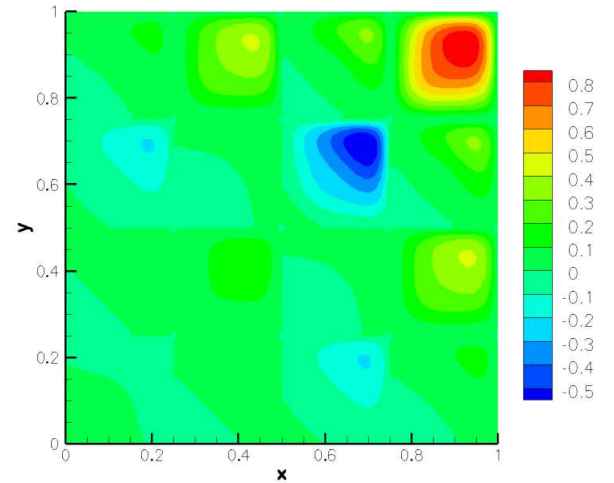

(a) $2 \mathrm{D}$ view

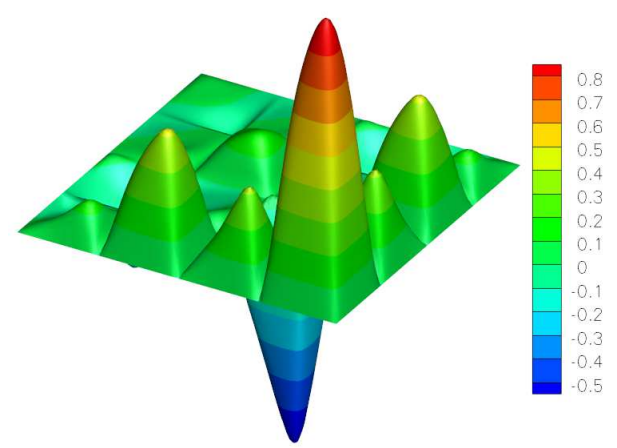

(b) 3D view

Figure 19: Convection-diffusion equation. Internal residual error, $u_{\text {bub }}^{\prime}$.

The total error is obtained summing both $u_{\text {bub }}^{\prime}$ and $u_{\text {poll }}^{\prime}$. Both the reference and the estimated error are very similar as can be seen in Fig. 21, even in this case in which the FEM solution is unstable.

\subsubsection{Error estimation for the SUPG method}

The SUPG solution [13] is obtained via Eq. (7) with a $8 \times 8$ mesh with bilinear quadrilaterals. Now, the numerical solution is stable in contrast with the Galerkin solution, see Fig. 22. 


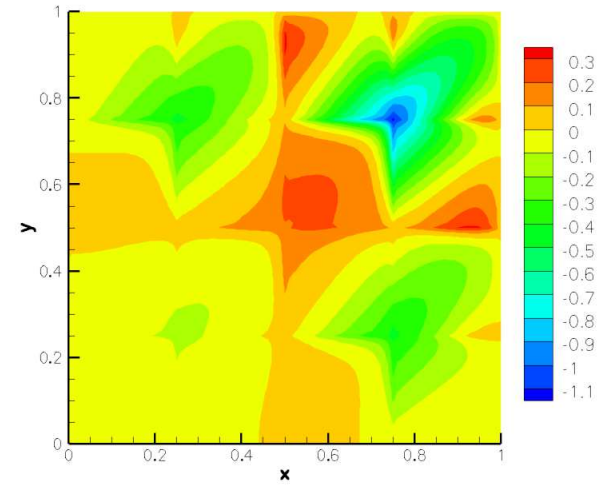

(a) $2 \mathrm{D}$ view

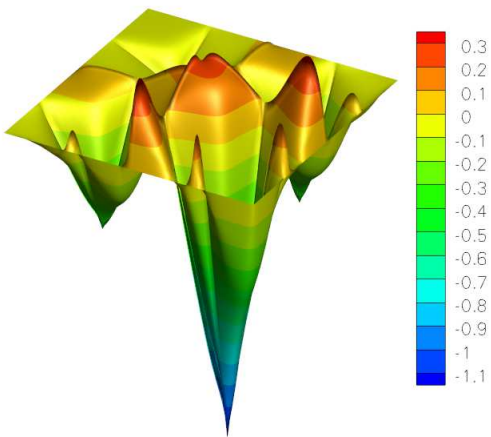

(b) $3 \mathrm{D}$ view

Figure 20: Convection-diffusion equation. Inter-element error, $u_{\text {poll }}^{\prime}$.

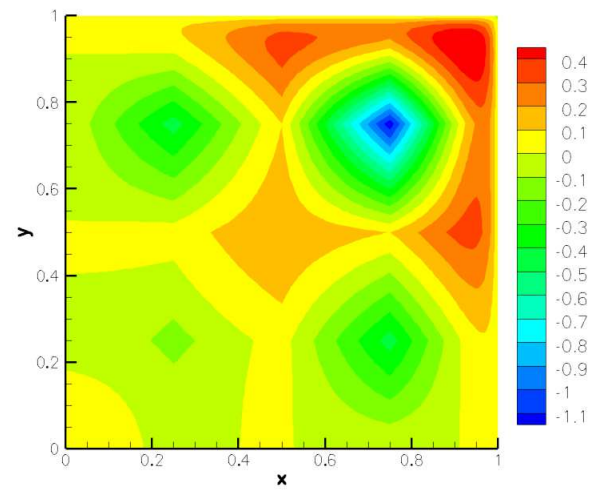

(a) Reference error

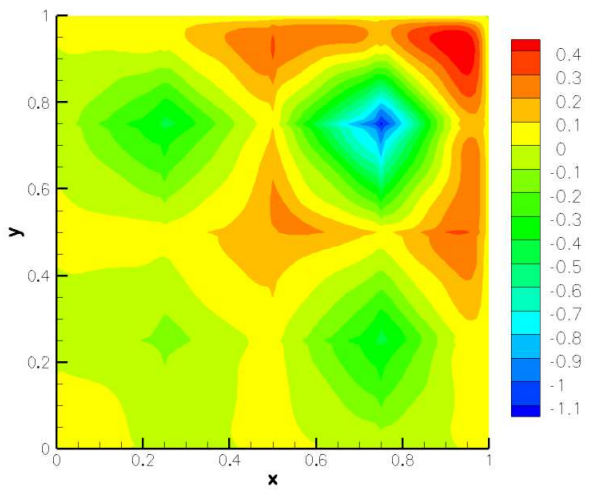

(b) Estimated error

Figure 21: Convection-diffusion equation. Reference and estimated error.

Once the SUPG solution is obtained, the stabilized solution is identified with the coarse scales, $\bar{u}$. Both terms of the error estimation are computed following the same steps as the latter case. Fig. 23 depicts the internal residual error, $u_{\text {bub }}^{\prime}$, and the inter-element error, $u_{\text {poll }}^{\prime}$. Summing both components the error estimation is achieved.

The error estimation and the reference error are represented in Fig. 24 and they are very similar. Since the SUPG solution is a stable solution, the error is practically zero in almost all the domain except in the boundary 


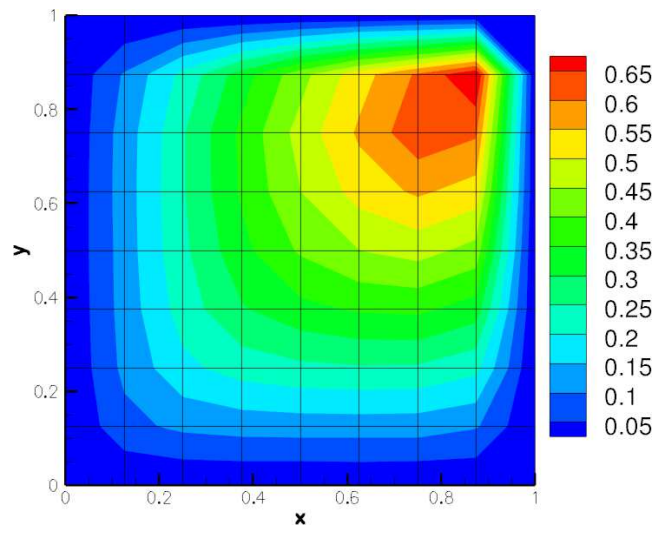

Figure 22: Convection-diffusion equation. SUPG solution.

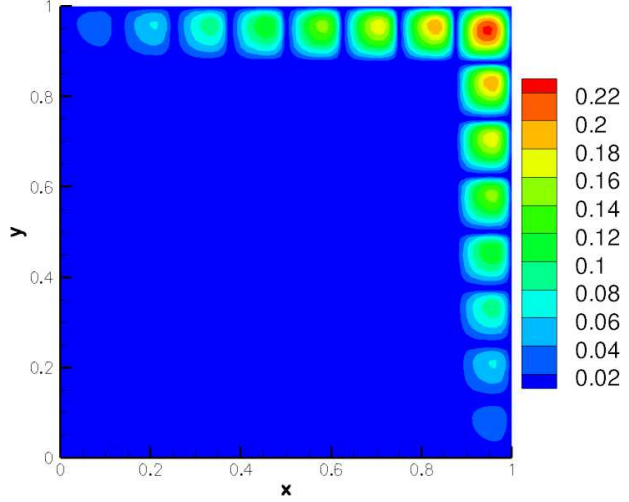

(a) $u_{\text {bub }}^{\prime}$

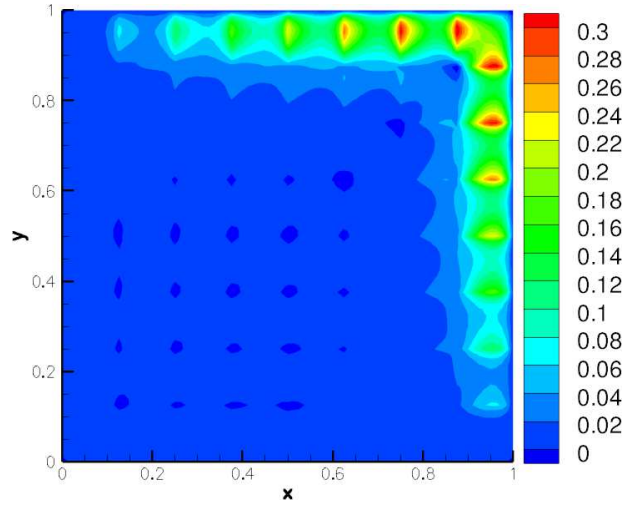

(b) $u_{\text {poll }}^{\prime}$

Figure 23: Convection-diffusion equation. Internal residual error and inter-element error for $\kappa=0.03$ and $\boldsymbol{a}=(1,1)$ with $f=1$ and SUPG solution

layer.

Remark 6. It can be appreciated that the SUPG error pollution has a local character. This feature can be exploited to develop feasible VMS explicit error estimators [20, 21].

\section{Adaptive refinement mesh using the error estimation}

Once the error estimation is obtained, this information can be used in an adaptive mesh refinement process. The convergence theory establishes that the error scales with the size of the element as [5], 


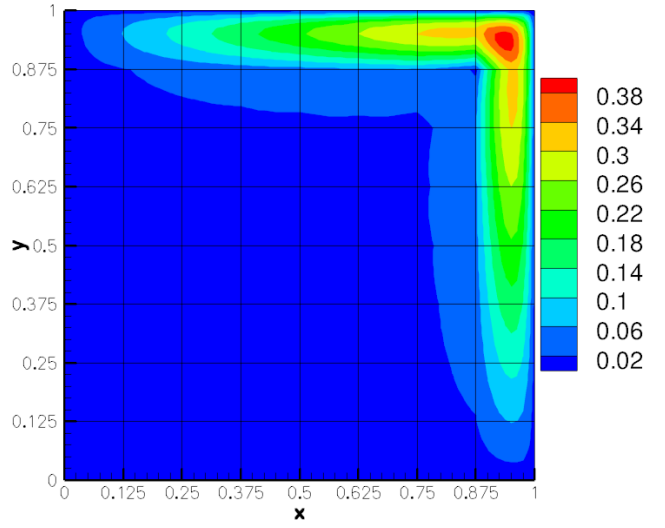

(a) Reference error

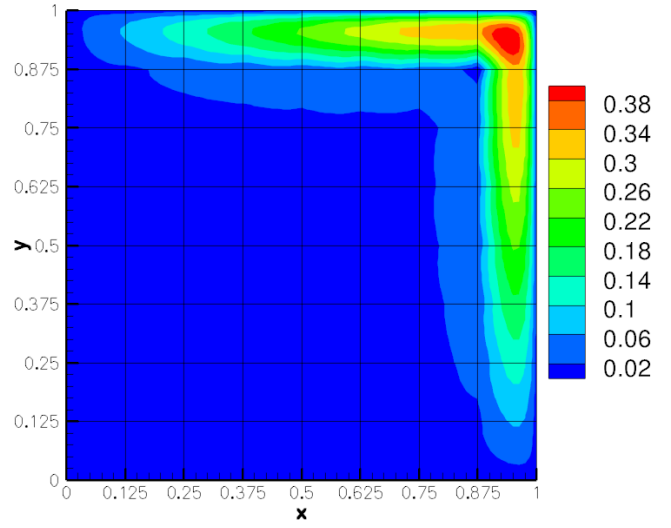

(b) Estimated error

Figure 24: Convection-diffusion equation. Reference and estimated error for $\kappa=0.03$ and $\boldsymbol{a}=(1,1)$ with $f=1$ and SUPG solution

$$
\left\|u^{\prime}\right\|_{L^{\infty}(\Omega)} \approx C \cdot h^{k+1}
$$

where $C$ is independent of the element size, $h$, and provided that the solution is sufficiently smooth. For linear or bilinear elements employed, Eq. (44) reads,

$$
\left\|u^{\prime}\right\|_{L^{\infty}(\Omega)} \approx C \cdot h^{2}
$$

Adaptive mesh refinement can be carried out controlling the maximum error. To do it, we can evaluate the error at the center of the element and/or at the center of the edges and/or at the nodes. These points are called control points.

The refinement is an iterative process. To define the refinement strategy [17], in each iteration we have to decide the new element size as a function of :

i The estimated error at the element control points

ii The tolerance error, which is introduced by the user

iii The current size of the element

Thus, according to convergence theory, the new element size is defined as 


$$
\frac{h_{\text {new }}^{2}}{h_{\text {old }}^{2}}=\frac{\left\|u_{\text {tol }}^{\prime}\right\|_{L^{\infty}\left(\Omega^{e}\right)}}{\left\|u_{\text {old }}^{\prime}\right\|_{L^{\infty}\left(\Omega^{e}\right)}}
$$

where $h_{\text {new }}$ and $h_{\text {old }}$ are the new and old element sizes, respectively. $u_{\text {old }}^{\prime}$ and $u_{\text {tol }}^{\prime}$ are the estimated error and the desired error. The norm $\|\cdot\|_{L^{\infty}\left(\Omega^{e}\right)}$ is the maximum error measured in absolute value at the control point belonging to the $\Omega^{e}$. The refinement process has been performed with GiD software [16].

For the SUPG simulation that we have seen previously in Section 4.3, we have refined the mesh taking an initial $4 \times 4$ quadrilateral mesh.

We set an error tolerance, $\left\|u_{\text {tol }}^{\prime}\right\|_{L^{\infty}\left(\Omega^{e}\right)}=0.05$. In Table 1 , the number of elements, $n_{\text {els }}$, the number of nodes $n_{n p}$ and the pointwise error are shown for the four iterations that have been made to obtain the final mesh with the desired pointwise error.

\begin{tabular}{l|ccc|} 
& $n_{\mathrm{els}}$ & $n_{n p}$ & $\left\|u^{\prime}\right\|_{L^{\infty}}$ \\
\hline Iteration 1(*) & 16 & 25 & $3.509 \mathrm{e}-1$ \\
Iteration 2 & 110 & 132 & $1.658 \mathrm{e}-1$ \\
Iteration 3 & 363 & 405 & $7.832 \mathrm{e}-2$ \\
Iteration 4 & 854 & 922 & $4.974 \mathrm{e}-2$ \\
\hline
\end{tabular}

(*)Uniform $4 \times 4$ mesh

Table 1: Maximum pointwise error in each iteration.

The initial and refined meshes are represented in Fig. 25.

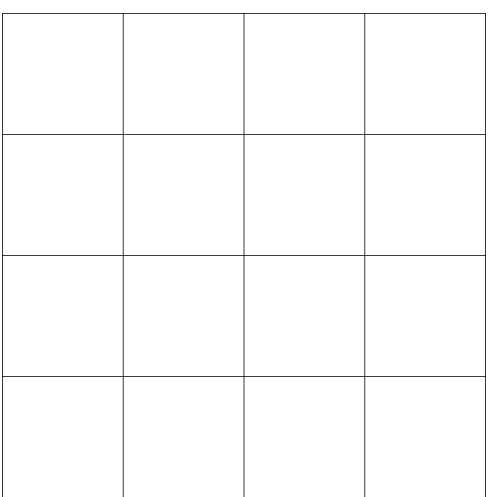

(a) Initial mesh

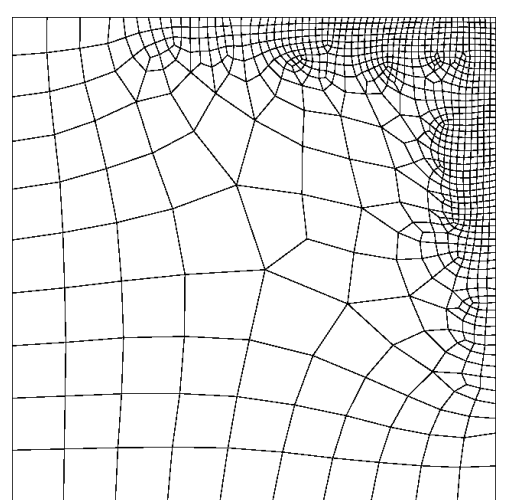

(b) Final mesh

Figure 25: Refine mesh for SUPG method with $\kappa=0.03, a_{x}=1$ and $a_{y}=1$. 


\section{Conclusions}

In this work, a methodology to obtain a posteriori the pointwise error based on the variational multiscale theory in combination with bubble functions and Green's functions has been presented. According to the nature of the error sources, the finite element error has been split into two components, $u_{\text {bub }}^{\prime}$ and $u_{\text {poll }}^{\prime}$. In particular, the error component $u_{\text {bub }}^{\prime}$ is related to the element internal residuals and has been modeled as a combination of bubble functions. The error component $u_{\text {poll }}^{\prime}$ is connected to the inter-element residuals (jumps) and the Neumann boundary conditions and it is computed making use of the fundamental solution or free-space Green's functions.

If residual-free bubbles and the problem Green's functions are employed, the formulation leads to an exact explicit method. However, in practice residual-free bubbles and classical Green's functions have complex analytical expressions or are hard to obtain. In this case, they can be substituted by bubble functions and free-space Green's functions, respectively. Then, the element interior residual must be projected into the bubble function space within each element and a global boundary integral equation must be solved to match the boundary conditions of the Green's functions. This study suggests that the fine-scale Green's functions can be decomposed into a combination of bubble functions and Green's functions along the element boundaries.

The theoretical formulation has been tested with numerical examples applied to the heat equation, the reaction-diffusion equation and the convectiondiffusion equation. The error has been analyzed for the standard Galerkin and SUPG methods. The numerical results show that the estimated error and the reference error are practically identical, demonstrating a good connection between the error sources and the error itself. Also, the numerical tests have shown that for stabilized methods the pollution error is confined within a local region, explaining the success of explicit residual-based VMS error estimators.

As an application, adaptive mesh refinements have been performed achieving meshes in which the users can control the pointwise error of the FEM solution.

\section{Acknowledgements}

The authors thank Ricardo Celorrio, Thomas J.R. Hughes, Matthias Taus and Fernando Lizarraga for evincing interest in this work and for helpful 
conversations.

This work has been partially funded by the Ministerio de Educación, Cultura y Deporte under FPU grant AP210-2073, the Ministerio de Economía y Competitividad under contract MAT2013-46467-C4-3-R and Gobierno de Aragón and FEDER funding from the European Union (Grupo Consolidado de Mecanica de Fluidos Computacional T21). 


\section{Appendix A. Internal residual error obtained by fine-scale Green's functions}

The internal residual error can be computed as

$$
u_{\text {bub }}^{\prime}(\boldsymbol{x})=-\int_{\widetilde{\Omega}} g^{\prime}(\boldsymbol{x}, \boldsymbol{y})(\mathcal{L} \bar{u}-f)(\boldsymbol{y}) \mathrm{d} \Omega
$$

The fine-scale Green's function, $g^{\prime}(\boldsymbol{x}, \boldsymbol{y})$, can be considered as the classic Green's functions in which the coarse scales are removed. Hughes and Sangalli obtained the general expression for the fine-scale Green's function

$$
\mathcal{G}^{\prime}=\mathcal{G}-\mathcal{G} \mathcal{P}^{T}\left(\mathcal{P G} \mathcal{P}^{T}\right)^{-1} \mathcal{P G}
$$

where $\mathcal{G}$ is the classical Green's function operator which represents the inverse of the differential operator, $\mathcal{L}^{-1}$. $\mathcal{P}$ is an orthogonal projector, $\mathcal{P}: \mathcal{S} \rightarrow \overline{\mathcal{S}}$ such that $\mathcal{P} v=\bar{v}$ and $\mathcal{P} v^{\prime}=0 \forall v^{\prime} \in \mathcal{S}^{\prime}$.

The suitable projector to obtain the fine-scale Green's function is the one associated with $H^{1}$-seminorm. For the heat equation, the $H^{1}$-projection is the same as the Galerkin projection.

Fig. A.26 shows the fine-scale Green's function for a $5 \times 5$ mesh. It is observed that $g^{\prime}(\boldsymbol{x}, \boldsymbol{y})$ is mostly local and is different from zero on the element boundary.

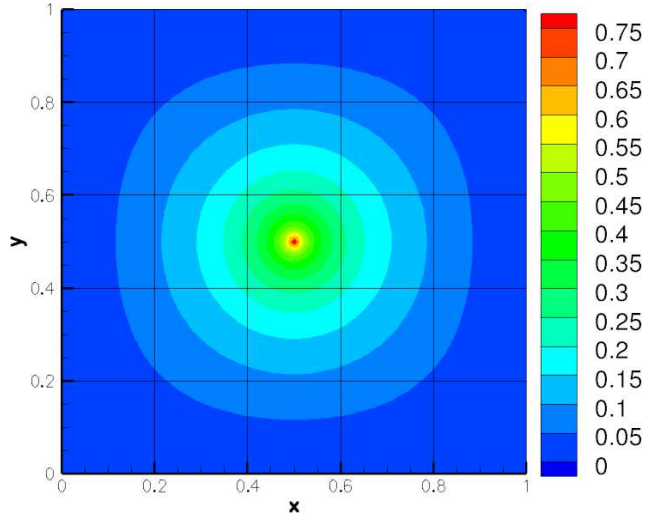

(a) $g(\boldsymbol{x}, \boldsymbol{y})$

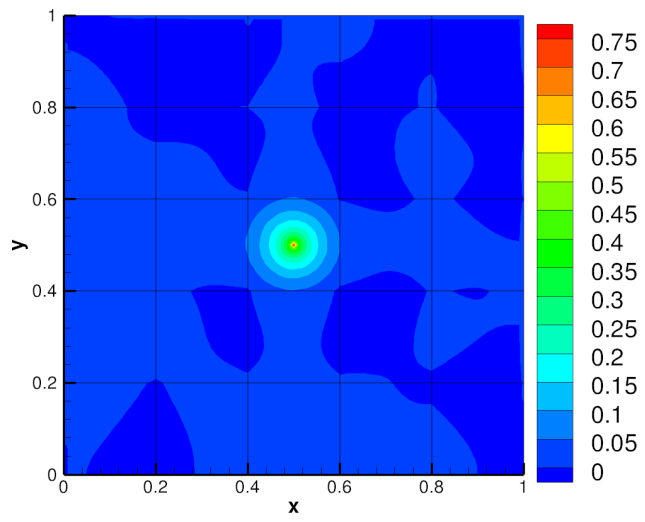

(b) $g^{\prime}(\boldsymbol{x}, \boldsymbol{y})$

Figure A.26: Heat equation. Green's function and fine-scale Green's function for $\boldsymbol{y}=$ $(0.5,0.5)$ in a $5 \times 5$ mesh. 
On the element boundary, Green's functions, $g(\boldsymbol{x}, \boldsymbol{y})$, are defined such that they are zero on the element boundary. These functions are called element Green's functions $g^{e}(\boldsymbol{x}, \boldsymbol{y})$ since their support is an element.

Thus, the error can be approximated as

$$
u_{\text {bub }}^{\prime}(\boldsymbol{x})=-\int_{\Omega^{e}} g^{e}(\boldsymbol{x}, \boldsymbol{y})(\mathcal{L} \bar{u}-f)(\boldsymbol{y}) \mathrm{d} \Omega
$$

Then, the internal residual error, $u_{\text {bub }}^{\prime}$, can be estimated by the same way as in 1D problems [28] via residual-free bubbles. Approximating by Taylor series and neglecting the second order terms

$$
\begin{aligned}
u_{\mathrm{bub}}^{\prime}(\boldsymbol{x}) & \approx u_{\mathrm{bub}}^{\prime}\left(\boldsymbol{c}_{i}\right)+\left.\nabla u_{\mathrm{bub}}^{\prime}(\boldsymbol{x})\right|_{\boldsymbol{x}=\boldsymbol{c}_{i}} \cdot\left(\boldsymbol{x}-\boldsymbol{c}_{i}\right) \\
& =b_{0}(\boldsymbol{x})(f-\mathcal{L} \bar{u})\left(\boldsymbol{c}_{i}\right)+\left.b_{y_{1}}^{e}(\boldsymbol{x}) \frac{d(f-\mathcal{L} \bar{u})}{d y_{1}}\right|_{\boldsymbol{y}=\boldsymbol{c}_{i}}+\left.b_{y_{2}}^{e}(\boldsymbol{x}) \frac{d(f-\mathcal{L} \bar{u})}{d y_{2}}\right|_{\boldsymbol{y}=\boldsymbol{c}_{i}}
\end{aligned}
$$

with $\boldsymbol{x}=\left(x_{1}, x_{2}\right)$ and $\boldsymbol{y}=\left(y_{1}, y_{2}\right)$ and $\boldsymbol{c}_{i}$ represent the coordinates of the element centroid. The residual-free bubble functions are defined as

$$
\begin{aligned}
b^{e}(\boldsymbol{x}) & =\int_{\Omega^{e}} g^{e}(\boldsymbol{x}, \boldsymbol{y}) \mathrm{d} \Omega_{y} \\
b_{y 1}^{e}(\boldsymbol{x}) & =\int_{\Omega^{e}} g^{e}(\boldsymbol{x}, \boldsymbol{y})\left(y_{1}-c_{i, 1}\right) \mathrm{d} \Omega_{y} \\
b_{y 2}^{e}(\boldsymbol{x}) & =\int_{\Omega^{e}} g^{e}(\boldsymbol{x}, \boldsymbol{y})\left(y_{2}-c_{i, 2}\right) \mathrm{d} \Omega_{y}
\end{aligned}
$$

\section{References}

[1] Ainsworth, M., Allendes, A., Barrenechea, G.R., Rankin, R.: Fully computable a posteriori error bounds for stabilised fem approximations of convection-reaction-diffusion problems in three dimensions. International Journal for Numerical Methods in Fluids 73(9), 765-790 (2013)

[2] Ainsworth, M., Oden, J.T.: A posterior error estimation in finite element analysis. John Wiley \& Sons (2000)

[3] Ainsworth, M., Rankin, R.: Realistic computable error bounds for three dimensional finite element analyses in linear elasticity. Computer Methods in Applied Mechanics and Engineering 200(21), 1909-1926 (2011) 
[4] Araya, R., Behrens, E., Rodriguez, R.: An adaptive stabilized finite element scheme for the advection-reaction-diffusion equation. Applied Numerical Mathematics 54, 491-503 (2005)

[5] Babuska, I., Strouboulis, T.: The finite element method and its reliability. Oxford university press (2001)

[6] Babuška, I., Strouboulis, T., Gangaraj, S., Upadhyay, C.: Pollution error in the h-version of the finite element method and the local quality of the recovered derivatives. Computer Methods in Applied Mechanics and Engineering 140(1), 1-37 (1997)

[7] Babuška, I., Strouboulis, T., Gangaraj, S.K.: Guaranteed computable bounds for the exact error in the finite element solution part i: Onedimensional model problem. Computer methods in applied mechanics and engineering 176(1), 51-79 (1999)

[8] Babuška, I., Strouboulis, T., Upadhyay, C., Gangaraj, S.: A posteriori estimation and adaptive control of the pollution error in the h-version of the finite element method. International journal for numerical methods in engineering 38(24), 4207-4235 (1995)

[9] Banerjee, P.K., Butterfield, R.: Boundary element methods in engineering science, vol. 17. McGraw-Hill London (1981)

[10] Brebbia, C.A., Dominguez, J.: Boundary elements: an introductory course. WIT press (1996)

[11] Brezzi, F., Franca, L., Hughes, T., Russo, A.: $b=\int g$. Comput. Meth. Appl. Mech. Engrng. 145, 329-339 (1997)

[12] Brezzi, F., Marini, L.: Augmented spaces, two-level methods, and stabilizing subgrids. International journal for numerical methods in fluids 40(1-2), 31-46 (2002)

[13] Brooks, A., Hughes, T.: Streamline upwind/petrov-galerkin formulations for convection dominated flows with particular emphasis on the incompressible navier-stokes equations. Comput. Meth. Appl. Mech. Engrng. 32, 199-259 (1982) 
[14] Dari, E., Durán, R.G., Padra, C.: Maximum norm error estimators for three-dimensional elliptic problems. SIAM Journal on Numerical Analysis 37(2), 683-700 (1999)

[15] Díez, P., Huerta, A.: A posteriori error estimation for standard finite element analysis. Comput. Methods Appl. Mech. Engrng. 163, 141-157 (1998)

[16] GiD: Personal Pre and Post Processor: URL: http://www.gidhome.com. International Center for Numerical Methods in Engineering (CIMNE) (2010)

[17] Hauke, G., Doweidar, M.H., Fuentes, S.: Mesh adaptivity for the transport equation led by variational multiscale error estimators. International Journal for Numerical Methods in Fluids 69(12), 1835-1850 (2012)

[18] Hauke, G., Doweidar, M.H., Fuster, D., Gomez, A., Sayas, J.: Application of variational a-posteriori multiscale error estimation to higherorder elements. Computational Mechanics 38, 382-389 (2006)

[19] Hauke, G., Doweidar, M.H., Miana, M.: Proper intrinsic scales for aposteriori multiscale error estimation. Comput. Meth. Appl. Mech. Engrng 195, 3983-4001 (2006)

[20] Hauke, G., Fuster, D., Doweidar, M.H.: Variational multiscale aposteriori error estimation for the multi-dimensional transport equation. Comput. Meth. Appl. Mech. Engrg. 197, 2701-2718 (2008)

[21] Hauke, G., Fuster, D., Lizarraga, F.: Variational multiscale a posteriori error estimation for systems: The Euler and Navier-Stokes equations. Computer Methods in Applied Mechanics and Engineering 283, 1493$1524(2015)$

[22] Hauke, G., Irisarri, D.: Variational multiscale a posteriori error estimation for systems. application to linear elasticity. Computer Methods in Applied Mechanics and Engineering 285, 291-314 (2015)

[23] Huerta, A., Diez, P.: Error estimation including pollution assessment for nonlinear finite element analysis. Computer Methods in Applied Mechanics and Engineering 181(1), 21-41 (2000) 
[24] Hughes, T.: Multiscale phenomena: Green's functions, the Dirichlet-toNeumann formulation, subgrid scale models, bubbles and the origins of stabilized methods. Comput. Meth. Appl. Mech. Engrng. 127, 387-401 (1995)

[25] Hughes, T.: The finite element method:Linear static and dynamic finite element analysis. Dover Publications (2000)

[26] Hughes, T., Feijoo, G., Mazzei, L., Quincy, J.: The variational multiscale method: A paradigm for computational mechanics. Comput. Meth. Appl. Mech. Engrg. 166, 3-24 (1998)

[27] Hughes, T., Sangalli, G.: Variational multiscale analysis: the fine-scale Green's function, projection, optimization, localization and stabilized methods. SIAM J. Numer. Anal. 45(2), 539-557 (2007)

[28] Irisarri, D., Hauke, G.: Pointwise error estimation for the transport equation based on variational multiscale method. Int. J. Comp. Meth. submitted

[29] Irisarri, D., Hauke, G.: Variational multiscale a posteriori error estimation for 2nd and 4th-order ODEs. International Journal of Numerical Analysis \& Modeling 12(3) (2015)

[30] Ladevèze, P., Leguillon, D.: Error estimate procedure in the finite element method and applications. SIAM J. Numer. Anal. 20, 485-509 (1983)

[31] Ladevèze, P., Maunder, E.: A general method for recovering equilibrating element tractions. Computer Methods in Applied Mechanics and Engineering 137(2), 111-151 (1996)

[32] Larson, M.G., Malqvist, A.: Adaptive variational multiscale methods based on a posteriori error estimation: Energy norm estimates for elliptic problems. Comput. Methods Appl. Mech. Engrg. 196, 2313-2324 (2007)

[33] Larsson, F., Díez, P., Huerta, A.: A flux-free a posteriori error estimator for the incompressible Stokes problem using a mixed fe formulation. Computer Methods in Applied Mechanics and Engineering 199(37), 2383-2402 (2010) 
[34] Masud, A., Truster, T., L.A.Bergman: A variational multiscale a posteriori error estimation method for mixed form of nearly incompressible elasticity. Comput. Methods Appl. Mech. Engrg. 200, 3453-3481 (2011)

[35] Nochetto, R.H.: Pointwise a posteriori error estimates for elliptic problems on highly graded meshes. Mathematics of computation 64(209), $1-22(1995)$

[36] Oden, J.T., Feng, Y.: Local and pollution error estimation for finite element approximations of elliptic boundary value problems. Journal of computational and Applied Mathematics 74(1), 245-293 (1996)

[37] Ohnimus, S., Stein, E., Walhorn, E.: Local error estimates of FEM for displacements and stresses in linear elasticity by solving local Neumann problems. International Journal for Numerical Methods in Engineering 52(7), 727-746 (2001)

[38] Parés, N., Díez, P., Huerta, A.: Subdomain-based flux-free a posteriori error estimators. Comput. Methods Appl. Mech. Engrng. 195, 297-323 (2006)

[39] Prudhomme, S., Nobile, F., Chamoin, L., Oden, J.: Analysis of a subdomain-based error estimator for finite element approximations of elliptic problems. Numerical Methods for Partial Differential Equations 20(2), 165-192 (2004)

[40] Prudhomme, S., Oden, J.T.: On goal-oriented error estimation for elliptic problems: application to the control of pointwise errors. Comput. Methods Appl. Mech. Engrng. 176, 313-331 (1999)

[41] Roos, H.G., Stynes, M., Tobiska, L.: Robust numerical methods for singularly perturbed differential equations: convection-diffusion-reaction and flow problems, vol. 24. Springer Science \& Business Media (2008)

[42] Song, L., Hou, Y., Zheng, H.: Adaptive variational multiscale method for the Stokes equations. International Journal for Numerical Methods in Fluids 71(11), 1369-1381 (2013)

[43] Stein, E., Rüter, M., Ohnimus, S.: Error-controlled adaptive goaloriented modeling and finite element approximations in elasticity. Computer methods in applied mechanics and engineering 196(37), 3598-3613 (2007) 